\title{
Radiosensitization effect of hsa-miR-138-2-3p on human laryngeal cancer stem cells
}

\author{
Ying Zhu ${ }^{1}$, Li-Yun Shi ${ }^{2}$, Yan-Min Lei ${ }^{3}$, Yan-Hong Bao ${ }^{3}$, Zhao-Yang Li ${ }^{4}$, Fei Ding ${ }^{4}$, Gui-Ting Zhu ${ }^{4}$, Qing- \\ Qing Wang ${ }^{5}$, Chang-Xin Huang ${ }^{\text {Corresp. } 4}$ \\ ${ }^{1}$ First Affiliated Hospital, Zhejiang Chinese Medical University, Hangzhou, China \\ 2 Department of Immunology, School of Medical and Life Science, Nanjing University of Chinese Medicine, Nanjing, China \\ 3 Second Clinical Medical College, Zhejiang Chinese Medical University, Hangzhou, China \\ 4 Department of Oncology, Affiliated Hospital with Hangzhou Normal University School of Medicine, Hangzhou, China \\ 5 Institute of Immunology, Zhejiang University, Hangzhou, China \\ Corresponding Author: Chang-Xin Huang \\ Email address: hcx588@hotmail.com
}

Background Treatment that targets to cancer stem cells plays an important role in controlling and eliminating of tumor initiation, development, progression, and chemotherapy/radiotherapy resistance. In our previous study, we cultured and harvested human laryngeal cancer stem cells (CSCs), applied microRNA biochips to screen differentially expressed miRNAs that were related to radiation tolerance in irradiated human laryngeal CSCs. According to the predicted genes and pathways of differential miRNAs target, hsa-miR-138-2-3p that was down-regulated expression under radiation, was thought to play a key role in enhancing the radio-sensitivity in human laryngeal squamous cancer stem cells. Method To investigate the radiational enhancement of hsamiR-138-2-3p, we transfected hsa-miR-138-2-3p mimics that were synthesized based on the sequences of hsa-miR-138-2-3p in vitro into human laryngeal CSCs (Hep-2, M2e, and TU212 cell lines) to make hsa-miR-138-2-3p overexpressed, and the tumorous specialities of CSCs, like cell proliferation, invasion, apoptosis, cell cycle arrest, and DNA damage were evaluated by CCK-8 assay, clone formation assay, invasion assay, flow cytometry, and comet assay. Furthermore, we explored the signal transduction pathways that regulated the cancer stem cell initiation, development, invasion, apoptosis and cell cycle arrest, which were controlled by hsa-miR-138-2-3p. Result Overexpressed hsa-miR-138-2-3p played an key role in many anti-cancer biological process in human laryngeal CSCs: (1) decreased laryngeal CSCs proliferation and invasion in response to radiotherapy; (2) increased the proportion of early and late apoptosis in laryngeal CSCs after radiation, raised G1 phase arrest in laryngeal CSCs after radiation, and decreased the proportion of S stage cells of cell cycle that were related to radio-resistance in laryngeal CSCs; (3) downregulated the expression of $\beta$-catenin in Wnt signal pathway that was related to the 
tolerance of laryngeal CSCs to radiotherapy; (4)down-regulated the expression of YAP1 in Hippo signal pathway that regulated cell proliferation, invasion and apoptosis; (5)upregulated the expression of p38 and JNK1 in MAPK signal pathway that was concerned to radio-sensitivity. Conclusion In the present study, it was found that hsa-miR-138-2-3p regulated Wnt/ß-catenin pathway, Hippo/YAP1 pathway, and MAPK/p38/JNK1 pathway that were involved in cell proliferation, invasion, apoptosis, cell cycle arrest, and radioresistance and radio-sensitivity in laryngeal CSCs. These results will be useful for a better understanding of cell biology of hsa-miR-138-2-3p in laryngeal CSCs, and serving hsa-miR138-2-3p as a promising biomarker and target for diagnosis and novel anti-cancer therapy for laryngeal cancers. 
1 Radiosensitization effect of hsa-miR-138-2-3p on

2

3

4

5 1: First Affiliated Hospital, Zhejiang Chinese Medical University, Hangzhou, China.

6 2: Department of Immunology, School of Medical and Life Science, Nanjing University of Chinese

7 Medicine, Nanjing, China.

8 3: Second Clinical Medical College, Zhejiang Chinese Medical University, Hangzhou, China.

9 4: Department of Oncology, Affiliated Hospital with Hangzhou Normal University School of Medicine,

10 Hangzhou, China.

11 5: Institute of Immunology, Zhejiang University, Hangzhou, China.

12 \#: Contributed equally

$13{ }^{*}$ Correspondence to: Chang-Xin Huang MD, PhD, Professor

14 Tel: +86-571-88358068; Fax: +86-571-88021730

15 Email: hcx588@hotmail.com

\section{hcx588@tom.com}

\section{Abstract}

Background Treatment that targets to cancer stem cells plays an important role in controlling and eliminating of tumor initiation, development, progression, and chemotherapy/radiotherapy resistance. In our previous study, we cultured and harvested human laryngeal cancer stem cells (CSCs), applied microRNA biochips to screen differentially expressed miRNAs that were related to radiation tolerance in irradiated human laryngeal CSCs. According to the predicted genes and pathways of differential miRNAs target, hsa-miR-138-2-3p that was down-regulated under radiation, was thought to play a key role in enhancing the radio-sensitivity in human laryngeal squamous cancer stem cells.

Method To investigate the radiational enhancement of hsa-miR-138-2-3p, we transfected hsa-miR$138-2-3 p$ mimics that were synthesized based on the sequences of hsa-miR-138-2-3p in vitro into 
27 human laryngeal CSCs (Hep-2, M2e, and TU212 cell lines) to make hsa-miR-138-2-3p 28 overexpressed, and the tumorous specialities of CSCs, like cell proliferation, invasion, apoptosis, cell cycle arrest, and DNA damage were evaluated by CCK-8 assay, clone formation assay, invasion assay, flow cytometry, and comet assay. Furthermore, we explored the signal transduction pathways that regulated the cancer stem cell initiation, development, invasion, apoptosis and cell cycle arrest, which were controlled by hsa-miR-138-2-3p.

Result Overexpressed hsa-miR-138-2-3p played an key role in many anti-cancer biological process in human laryngeal CSCs: (1) decreased laryngeal CSCs proliferation and invasion in response to radiotherapy; (2) increased the proportion of early and late apoptosis in laryngeal CSCs after radiation, raised G1 phase arrest in laryngeal CSCs after radiation, and decreased the proportion of S stage cells of cell cycle that were related to radio-resistance in laryngeal CSCs; (3) down-regulated the expression of $\beta$-catenin in Wnt signal pathway that was related to the tolerance of laryngeal CSCs to radiotherapy; (4)down-regulated the expression of YAP1 in Hippo signal pathway that regulated cell proliferation, invasion and apoptosis; (5)up-regulated the expressions of p38 and JNK1 in MAPK signal pathway that was concerned to radio-sensitivity.

42 Conclusion In the present study, it was found that hsa-miR-138-2-3p regulated Wnt/ 3 -catenin pathway, Hippo/YAP1 pathway, and MAPK/p38/JNK1 pathway that were involved in cell proliferation, 44 invasion, apoptosis, cell cycle arrest, and radio-resistance and radio-sensitivity in laryngeal CSCs.

45 These results will be useful for a better understanding of cell biology of hsa-miR-138-2-3p in laryngeal 46 CSCs, and serving hsa-miR-138-2-3p as a promising biomarker and target for diagnosis and novel 47 anti-cancer therapy for laryngeal cancers.

\section{Introduction}

49 Many studies have shown that biological characteristics of tumors, including radiation tolerance, are determined by tumor stem cells. Rycaj et al[1] showed that breast cancer and malignant glioma were tolerant to radiotherapy mainly due to the existence of cancer stem cells, which had powerful DNA repair capacity, defensive ability against reactive oxygen species and strong self-renewal capacity. 
53 Claudia et al[2] indicated that the plasticity of cancer stem cells would be beneficial under the stress

54 of various factors, and cancer stem cells (CSCs) were involved in reducing radio-sensitivity by 55 changing their structures to generate radiation-induced resistance. Selcuk et al[3] found that the 56 nature of radiation resistance of tumor cells were cancer stem cells that prevented cancer cell 57 apoptosis and enhanced the efficiency of DNA repair. Therapies target to CSCs are necessary for controlling and eliminating tumor cell growing, invasion, progression and radio-resistance. Yosuke et al [4] found that the removal of Brachyury that was related to cell proliferation, migration, invasion, radiation and chemotherapy resistance of Adenoid Cystic carcinoma CSCs, could improve tumor

61 stem cell radiation sensitivity. Wu et al[5] developed a model of CDK1 knock-out brain malignant 62 glioma and found that CDK1 contributed to enhance radiosensitivity of CSCs by inducing cell apoptosis. Cell cycle checkpoint kinases (CDK1 and CDK2) play key roles in DNA damage response in radiation and chemotherapy. MicroRNA (miRNA), a non-coding oligonucleotide single chain of $\sim 20$ t in length, was recently considered as the most important gene regulation factor in cancer cells, and may improve the radiosensitivity of tumor cells[6]. Jiang et al[21]found that increased expression of miR-17-92 cluster could enhance the resistance to radiation of lymphoma. Wu et al[22] demonstrated that MiR-148b could enhance radiosensitivity of non-Hodgkin's lymphoma cells by promoting apoptosis. However, the role of hsa-miR-138-2-3p in laryngeal CSCs was not reported before.

In our previous study[7], we used microRNA biochips to compare and screen the differential expression microRNAs in laryngeal CSCs in response to radiation stress. Based on the predicted genes and pathways of miRNA target, the expression profile of hsa-miR-138-2-3p that was downregulated significantly after radiation, was thought to play an important role in regulation of radiosensitivity in laryngeal squamous CSCs. In present study, we synthesized hsa-miR-138-2-3p in vitro and transfected it into three types of laryngeal CSCs(Hep-2, M2e, TU212) to make hsa-miR-138-2-3p overexpressed, and the tumorous specialities of CSCs, such as cell proliferation, invasion, apoptosis, cell cycle arrest, and DNA damage. Furthermore, we explored the signal transduction pathways that 
were involved in cell initiation, development, invasion, apoptosis and cell cycle arrest, which were regulated by hsa-miR-138-2-3p. These results will be useful for a better understanding of cell biology of hsa-miR-138-2-3p in laryngeal CSCs, and serving hsa-miR-138-2-3p as a promising biomarker and target for diagnosis and novel anti-cancer therapy for laryngeal cancers.

\section{Materials and Methods}

\section{Laryngeal cancer sphere culture}

85 Three human laryngeal squamous cancer cell lines, Hep-2, TU212 and M2e, were obtained from the 86 American Type Culture Collection (ATCC, USA). Serum supplement medium (SSM) contained 90\% 87 RPMI-1640 (Gibco, USA) and 10\% fetal bovine serum (Gibco, USA). Serum free medium (SFM) 88 contained DMEM/F12 (Gibco, USA); and $4 \mathrm{mg} / \mathrm{ml}$ heparin; $10 \mathrm{ng} / \mathrm{ml}$ basic fibroblast growth factor

89 (bFGF, Peprotech, USA), 20 ng/ml epidermal growth factor (EGF, Peprotech, USA); 25 mg/ml insulin; and $2 \mathrm{ml} 50 \times$ B27 supplement (Gibco, USA). Cells in exponential growth phase were washed with PBS (Gibco, USA) and digested with 0.25 trypsin/0.02\% ethylenediaminetetraacetic acid (EDTA, Gibco, USA), followed by resuspension in SFM at a concentration of $5 \times 10 E 5 \mathrm{cells} / \mathrm{ml}$. The medium was changed every 5 days in half amount. Each cell line was regularly observed to confirm its morphology and absence of mycoplasma contamination.

\section{Sorting of laryngeal CSCs based on cell surface marker expression}

96 The laryngeal cancer sphere of Hep-2, M2e and TU212, was digested, a single-cell suspension was 97 prepared and the cell number was counted before labeling. Cells were collected by centrifuge at 1000 98 rpm for 5 min and the cell pellets were resuspended in 90ul of PBS buffer per 10E7 total cells. 10ul of anti-human-CD133-FITC (AC-133-FITC, mouse IgG1, Miltenyi, Germany) were added. The samples were mixed well and incubated in the dark for $30 \mathrm{~min}$ at $4^{\circ} \mathrm{C}$ refrigerator. The analysis was performed with FACS caliber (BD, USA), and CD133 positive expression cells were investigated as laryngeal

102 CSCs.

\section{Hsa-miR-138-2-3p targets prediction}

104 In our earlier research [7], laryngeal CSCs were harvested and accepted to radiation stress. We 
105 applied microRNA biochips to identify and screen differential expression miRNAs, and more than 2-

106 fold up-regulation/down-regulation expression were considered as differential expressions.

107 Meaningful miRNAs were selected by targeted genes from Targetscan Human 6.2

108 (http://www.targetscan.org [10]) and miRanda (http://www.microrna.org/microrna/home.do [11]). The

109 sequences of miRNAs were inquired from miRBase (http://www.miRbase.org [12]). To understand

110 the targeted biological process, we applied starBase v2.0 (http://starbase.sysu.edu.cn/index.php)[13]

111 to analyze signal transduction pathways that were regulated by microRNAs from pathway databases

112 (e.g. GO, KEGG, BIOCARTA). Hsa-miR-138-2-3p mimics, nonsense oligonucleotides, and negative

113 control FAM oligonucleotides with fluorescence were synthesized in vitro (invitrogen, Shanghai,

114 China).

\section{Transient cell transfection}

116 Laryngeal CSCs (2X10E5 cells/ well) were plated in 12-well culture plates, and were transfected

117 equal volume with gradient concentrations of hsa-miR-138-2-3p mimics (conc: $50 \mathrm{nM}, 100 \mathrm{nM}, 150$

$118 \mathrm{nM}$ ). Nonsense oligonucleotides (conc: $100 \mathrm{nM}$ ), negative control FAM oligonucleotides (conc: 100

$119 \mathrm{nM}$ ), and PBS buffer with the same volume as hsa-miR-138-2-3p were transfected into laryngeal

120 CSCs. The hsa-miR-138-2-3p teams with gradient concentration were considered as experimental

121 team and were named as 50nM-TR, 100nM-TR, 150nM-TR respectively. Nonsense oligonucleotides

122 team, negative control FAM oligonucleotides team, and PBS buffer team were considered as control

123 teams, and were named as 100nMN-CR, FAM-CR, and PBS-CR. All the teams were added in

124 Entranster ${ }^{\mathrm{TM}}-\mathrm{R}$ transfection reagent (Engreen Biosystem, China) and mixed sufficiently, according to

125 the manufacturer's instructions. All teams were with the final concentrations of 50nM per well. After

126 mixing, all 12 -well culture plates were incubated for $6 \mathrm{~h}$ at $4^{\circ} \mathrm{C}$ refrigerator. The transfection efficiency

127 of hsa-miR-138-2-3p mimics and nonsense oligonucleotides were evaluated by the positive

128 expression of negative control FAM oligonucleotides by flow cyometry.

129 Irradiation

130 Laryngeal CSCs were irradiated by a linear accelerator with a 6-MV X ray. Culture plates were placed 
131 under a $15 \mathrm{~mm}$ tissue equivalent filler. The distance between filler and radiation source was $100 \mathrm{~mm}$.

132 Experimental teams and control teams were irradiated continuously at total does of 2 Gy each day, for

1332 days. $100 \mathrm{nM}-\mathrm{T}, 100 \mathrm{nMN}-\mathrm{C}, \mathrm{PBS}-\mathrm{C}$ teams were treated without radiation and were placed out the

134 range of radiation at the same time. All the three teams were served as control teams.

135 Proliferation assays

136 All the experimental and control teams were respectively resuspended in $0.1 \mathrm{ml} \mathrm{SFM}$ at a density of

1375000 cells/well in 96 -well microwell culture plates. Cell proliferation were analyzed at $0 \mathrm{~h}, 24 \mathrm{~h}, 48 \mathrm{~h}$

138 and $72 \mathrm{~h}$ after the second radiation by CCK-8 assay (Cell Counting Kit-8, Engreen Biosystem,

139 China)[14]. Viable cells were quantified by measuring absorbance at $450 \mathrm{~nm}$ absorption spectra in a

140 microplate reader, and were named as "A450 value".

\section{Apoptosis and cell cycle assay}

142 For apoptosis assay, 100nM-TR and 100nMN-CR of Hep-2, M2e, and TU212 CSCs were 143 resuspended in PBS buffer by the amount of cells 5000/ml. 195 ul cell suspension were mixed well 144 with 5 ul Annexin V-FITC and incubated at room temperature for $10 \mathrm{~min}$. Cells were washed with PBS 145 and resuspended in $190 \mathrm{ul}$ deliquated binding buffer, then $10 \mathrm{ul} 20 \mathrm{ug} / \mathrm{ml} \mathrm{PI}$ were added. Identified by 146 flow cytometry, cells were divided into four sections: Q1: Annexin V-FITC - PI +, was representative of 147 mechanical error; Q2: Annexin V-FITC+ PI+, was representative of late apoptosis or necrosis cells; 148 Q3: Annexin V-FITC - PI -, was representative of living cells; Q4: Annexin V-FITC+ PI-, was 149 representative of early apoptosis cells.

150 For cell cycle analysis, 100nM-TR and 100nMN-CR of Hep-2, M2e, and TU212 CSCs were washed 151 with cold PBS for three times, and then the cells were fixed in $70 \%$ ethanol at $-20^{\circ} \mathrm{C}$ for $12 \mathrm{~h}$. Following 152 the fixation, cells were washed with cold PBS and stained with 500ul PI at $37^{\circ} \mathrm{C}$ in dark for $30 \mathrm{~min}$. 153 Analyses were performed on flow cytometry.

\section{Invasion assays}

155 For Transwell migration assay, 12-well Transwell chambers containing 8 um pores were coated with 156700 ul Matrigel (BD, USA) at $-20^{\circ} \mathrm{C}$. According to the manufacturer's instructions[15], 100nM-TR and 
157 100nMN-CR of Hep-2, M2e, and TU212 CSCs were collected and resuspended in $1 \mathrm{ml}$ DMEM/F12 at

158 a density of 2 X10E5 cells/ $\mathrm{ml} .1 \mathrm{ml}$ cell suspension of six teams were added to the upper Transwell

159 chamber, and 600 ul SFM were added to the lower chamber. After $24 \mathrm{~h}$ of cell incubation, cells that

160 were cultured previously above the upper chambers, migrated to the other side of the upper

161 chambers. The reverse side of the upper chambers were fixed with $4 \%$ paraformaldehyde and

162 stained with crystal violet. The numbers of migrated cells on the reverse side were counted at least

163 five random microscopic fields by a light microscope at a magnification of 200X (Olympus, Japan).

\section{Clone formation assay}

165 100nM-TR, 100nMN-CR, and non-transfection teams of Hep-2, M2e, and TU212 CSCs that

166 proliferated at exponential growth phase, were digested and cultured at the density of 10E6/dish in 10

$167 \mathrm{~cm}$ petri dishes. After $24 \mathrm{~h}$ of culture, cells were treated with 0, 2, 4, 6, 8 Gy X-ray irradiation

168 respectively, and then were incubated for additional 14 days. After incubation, cells were washed with

169 PBS buffer for 3 times, and fixed with paraformaldehyde for $20 \mathrm{~min}$ and air dried overnight. Cells were

170 then washed with water and the colonies (> 50 cells) were counted for each culture dish by a light

171 microscope (Olympus, Japan).

\section{Comet assay}

173 100nM-TR and 100nMN-CR of Hep-2, M2e, and TU212 CSCs at equal volume of cell suspension

174 (4X10E5 cells) were mixed with $0.5 \%(\mathrm{w} / \mathrm{v})$ low melting agarose (LMA) in $0.01 \mathrm{M}$ PBS buffer

175 respectively. The mixture were pipetted on the frosted slides with pre-coating of normal melting

176 agarose $1 \%(\mathrm{w} / \mathrm{v})$. After the agarose solidified, another $100 \mu \mathrm{l}$ of $0.5 \%(\mathrm{w} / \mathrm{v})$ LMA were pipetted on the

177 slides and immersed in lysis buffer (2.5 M NaCl, $100 \mathrm{mM}$ EDTA, $10 \mathrm{mM}$ Tris-HCl buffer, $0.1 \%$ SDS

178 and $1 \%$ Triton $\mathrm{X}-100$ and $10 \%$ DMSO; $\mathrm{pH} 10.0$ ) for $120 \mathrm{~min}$ in dark at $4^{\circ} \mathrm{C}$ to lyse the cellular and

179 nuclear membranes. The slides were rinsed with unwinding buffer and transferred into an

180 electrophoresis tank containing unwinding buffer (3 M NaOH, $10 \mathrm{mM}$ EDTA; $\mathrm{pH}$ 13.0) for denaturing

181 the DNA followed by electrophoresis for 30 min with an electric current of $25 \mathrm{~V}$. The slides were

182 washed twice with neutralizing buffer $(0.4 \mathrm{M}$ Tris- $\mathrm{HCl}$; $\mathrm{pH} 7.5)$ for 10 min and ethanol treatment was 
183 done another $5 \mathrm{~min}$. Ethidium bromide $(20 \mathrm{mg} / \mathrm{ml}) 40 \mu \mathrm{l}$ were used to stain the slides and DNA

184 damage visualized using fluorescence microscope (Olympus, Japan). Appearance of 'comet' with

185 fragmented DNA (tail) being separated from undamaged nuclear DNA (head) was seen in damaged

186 cells and measurements were made by Comet Assay IV software to determine the tail movement (\%).

187 The results were expressed as percent tail movement.

188 Western blotting

189 The total protein of 100nM-TR, 100nMN-CR, and non-transfection teams of Hep-2, M2e, and TU212

190 CSCs were separated by $10 \%$ sodium dodecyl sulfate polyacrylamide gel electrophoresis (SDS-

191 PAGE) and transferred to membranes. The membranes were blocked in 5\% BSA, diluted in 1X TBS-

192 Tween for $1 \mathrm{~h}$ and then incubated overnight with anti-human $\beta$-catenin/YAP1/p38/JNK1 antibody

193 (Abcam, USA) according to the manufacturer's instructions. Primary antibody binding was detected

194 with secondary IgG-HRP antibodies goat anti-rabbit (Abcam, USA). Actin was used as control.

195 Images were captured on MicroChemi 4.2 (Eastwin, Israel).

\section{Statistical analysis}

197 Data are shown as mean \pm standard deviation $\left(\mathrm{x}^{ \pm} \pm \mathrm{s}\right)$. One-way and two-way ANOVA analyses were

198 applied to compare the sample means of test groups and control groups. The Fisher's exact test was

199 applied to compare the sample rates of test groups and control groups. All statistical analyses were

200 performed using SPSS 17.0 software (SPSS Inc., USA). P<0.05 was considered statistically 201 significant.

\section{Results}

\section{1. Hsa-miR-138-2-3p was selected to transfected into the laryngeal CSCs}

204 Based on our previous study [7], we applied Targetscan and Miranda to investigate target genes of 205 hsa-miR-138-2-3p, such as MAP3K11, CASP3 and HIF1AN, Which were involved in cell apoptosis, 206 radio-sensitivity, and cell cycle arrest (Table 1). 


\begin{tabular}{ccc}
\hline Target gene & $\begin{array}{c}\text { Representative } \\
\text { transcript }\end{array}$ & Gene name \\
\hline MAP3K11 & NM_002419 & mitogen-activated protein kinase kinase kinase 11 \\
ARHGEF3 & NM_001128615 & Rho guanine nucleotide exchange factor (GEF) 3 \\
HIF1AN & NM_017902 & hypoxia inducible factor 1, alpha subunit inhibitor \\
CASP3 & NM_004346 & caspase 3, apoptosis-related cysteine peptidase \\
ACVR2B & NM_001106 & activin A receptor, type IIB \\
\hline
\end{tabular}

The sequences of hsa-miR-138-2-3p were inquired from miRBase, and hsa-miR-138-2-3p mimics,

209 nonsense oligonucleotides, and negative control FAM oligonucleotides with fluorescence were

210 synthesized in vitro (invitrogen, Shanghai, China). The oligonucleotide sequences were listed in

211 Table 2.

212

Table 2. Sequence of synthesized oligonucleotides in vitro

\begin{tabular}{cc}
\hline Oligonucleotides & Sequence \\
\hline hsa-miR-138-2-3p mimics & 5' - GCUAUUUCACGACACCAGGGUU - 3' \\
nonsense oligonucleotides & 5'-AAGGCAAGCUGACCCUGAAGU-3' \\
Fluorescein FAM tag & 5'-UUCUCCGAACGUGUCACGUTT-3' \\
oligonucleotides & 3' - ACGUGACACGUUCGGAGAATT- 5' \\
\hline
\end{tabular}

213 To evaluate the transfection efficiency of hsa-miR-138-2-3p and nonsense oligonucleotides, the

214 FAM-CR teams of Hep-2, M2e, and TU212 cell lines that were transfected with FAM-labeled

215 oligonucleotide were digested and resuspended in PBS buffer. The percentages of FAM-positive

216 cells were identified by flow cytometry. As shown in Figure 1, the transfection efficiency of Hep-2,

217 M2e, and TU212 cell lines were $82.9 \%, 91.5 \%$ and $90.5 \%$ respectively, and it was indicated that hsa-

218 miR-138-2-3p and nonsense oligonucleotides were successfully transfected into the laryngeal CSCs

219 with high efficiency. 
220

221

222

223

224

225

226

227

228

229

230

231

232

233

234

235

236

237

238
A.

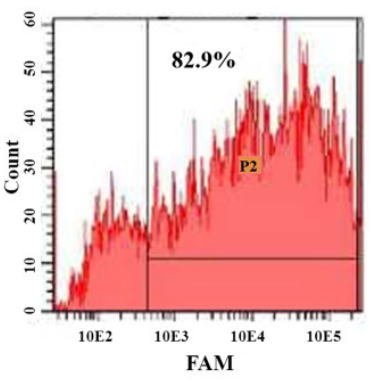

B.

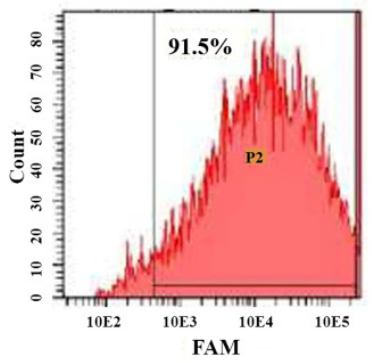

c.

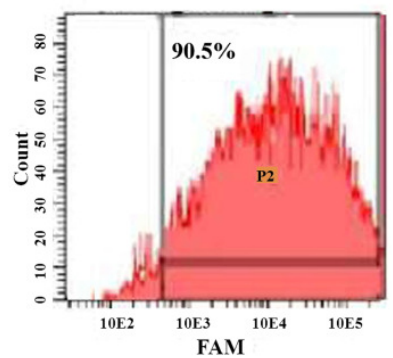

Figure 1. Cell transfection efficiency evaluated by Flow Cytometry.

Figure $1 \mathrm{~A}, 1 \mathrm{~B}$ and $1 \mathrm{C}$ showed the transfection efficiency of FAM-CR teams of Hep-2, M2e, and TU212 cell line respectively, and the percentages of FAM-positive cells (represented by "P2") were identified by flow cytometry. The transfection efficiency of Hep-2, M2e, and TU212 cell lines were $82.9 \%, 91.5 \%$ and $90.5 \%$ respectively, and it was indicated that hsa-miR-138-2-3p and nonsense oligonucleotides were successfully transfected into the laryngeal CSCs with high efficiency.

2. Overexpressed hsa-miR-138-2-3p was involved in various biological process to enhance the radiosensitivity of laryngeal CSCs

\subsection{Overexpressed hsa-miR-138-2-3p inhibited cell proliferation after radiation}

The cell proliferation rates of each team at $0,24,48$ and 72 hours after radiation were shown in Figure 2. From Figure $2 \mathrm{~A}$, we can infer that at $48 \mathrm{~h}$ after radiation, the cell proliferation rate of $50 \mathrm{nM}$ TR, 100nM-TR, and 150nM-TR were lower than 100nMN-CR and PBS-CR. But at 0h, 24h and 72h after radiation, the difference were not observed $(P>0.05)$. It was noted that at $48 \mathrm{~h}$ after radiation, the cell proliferation rate of $50 \mathrm{nM}-\mathrm{TR}, 100 \mathrm{nM}-\mathrm{TR}$, and $150 \mathrm{nM}-\mathrm{TR}$ were slower than $100 \mathrm{nMN}-\mathrm{CR}$ and PBS-CR, the differences were statistically significant $(P<0.001, P<0.001, P<0.001)$, and the inhibition capacity of 100nM-TR and 150nM-TR were stronger than 50nM-TR. While, from Figure 2B, it showed that without radiation, the differences of cell proliferation rate of $100 \mathrm{nM}-\mathrm{T}, 100 \mathrm{nMN}-\mathrm{C}$, and PBS-C were not statistically significant $(P>0.05)$. 


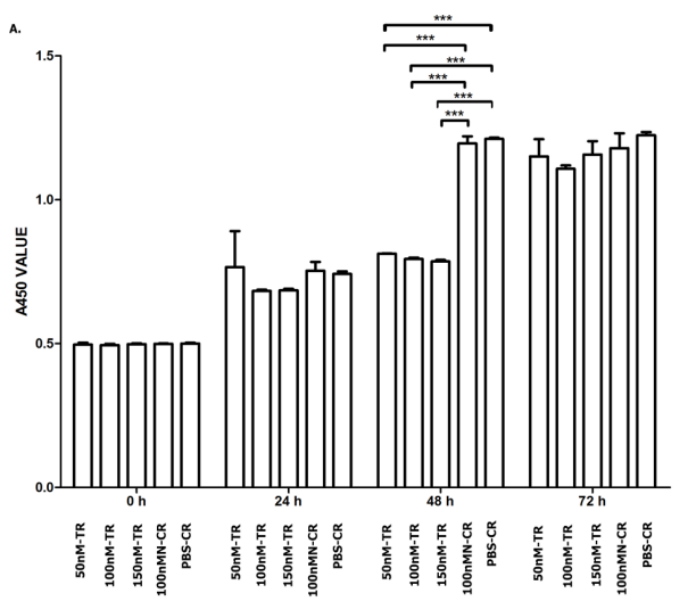

239

240

241

242 $(P<0.001)$ (Figure 3, Figure4).

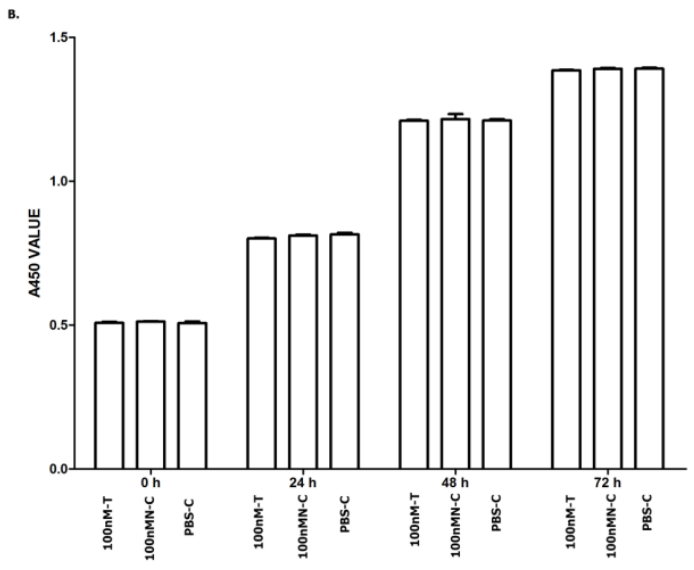

Incubation time after radation (hour)

Figure 2. Overexpressed hsa-miR-138-2-3p inhibit cell proliferation after radiation. Figure $2 \mathrm{~A}$ showed the comparison of cell proliferation among 50nM-TR, 100nM-TR, 150nM-TR, 100nMN-CR and PBS-CR of Hep-2 cell line after radiation. Figure 2B showed the comparison of cell proliferation among 100nM-T, 100nMN-C and PBS-C of Hep-2 cell line without radiation. The vertical and horizontal axis standed for A450 (absorbance at $450 \mathrm{~nm}$ absorption spectra) value and time after radiation respectively. From Figure $2 \mathrm{~A}$, we can infer that at $48 \mathrm{~h}$ after radiation, the cell proliferation rate of 50nM-TR, 100nM-TR, and 150nM-TR were lower than 100nMN-CR and PBS-CR. But at Oh, $24 \mathrm{~h}$ and $72 \mathrm{~h}$ after radiation, the difference were not observed $(P>0.05)$. It was noted that at $48 \mathrm{~h}$ after radiation, the cell proliferation rate of 50nM-TR, 100nM-TR, and 150nM-TR were slower than 100nMN-CR and PBS-CR, the differences were statistically significant $\left.{ }^{* * *} \mathrm{P}<0.001\right)$, and the inhibition capacity of $100 \mathrm{nM}-\mathrm{TR}$ and $150 \mathrm{nM}-\mathrm{TR}$ were stronger than 50nM-TR. While, from Figure 2B, it showed that without radiation, the differences of cell proliferation rate of 100nM-T, 100nMN-C, and PBS-C were not statistically significant $(P>0.05)$. Date are reported as mean $\pm S D$.

\subsection{Overexpressed hsa-miR-138-2-3p induced cell apoptosis after radiation}

To investigate whether the declined cell proliferation was due to the cell apoptosis, we used flow cytometry analyses to evaluate the effect of overexpressed hsa-miR-138-2-3p on promotion of cell apoptosis. We found that the proportion of early apoptosis and late apoptosis of Hep-2, M2e, and TU212 CSCs induced by transfection of hsa-miR-138-2-3p were larger than that induced by transfection of nonsense oligonucleotides, the differences between them were statistically significant 
A.

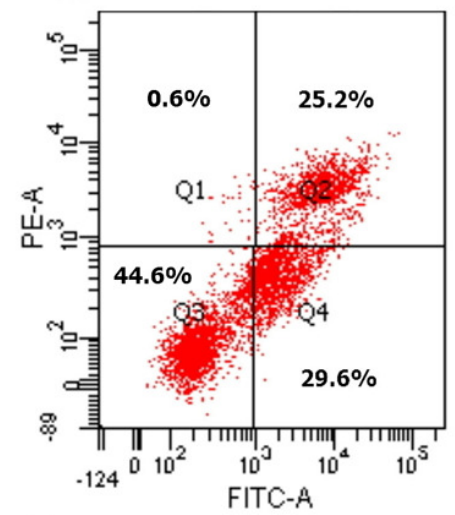

D.

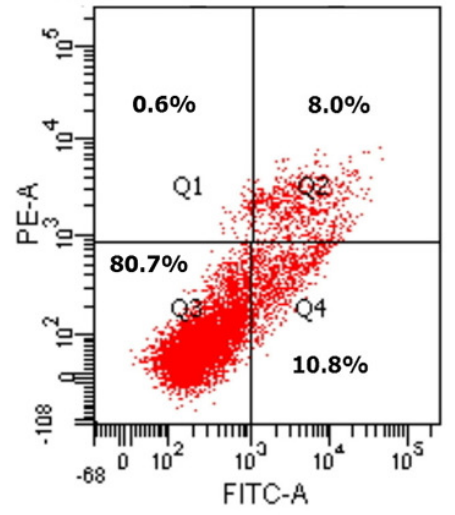

B.

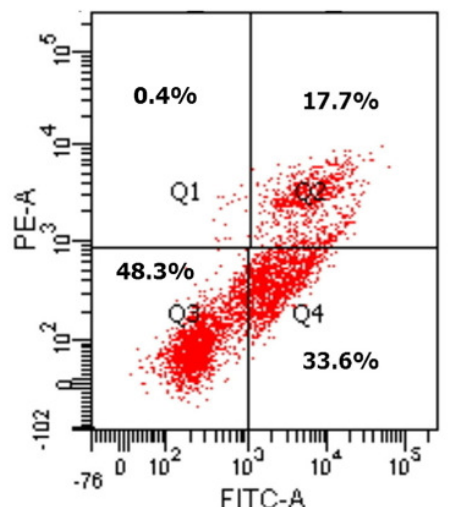

E.

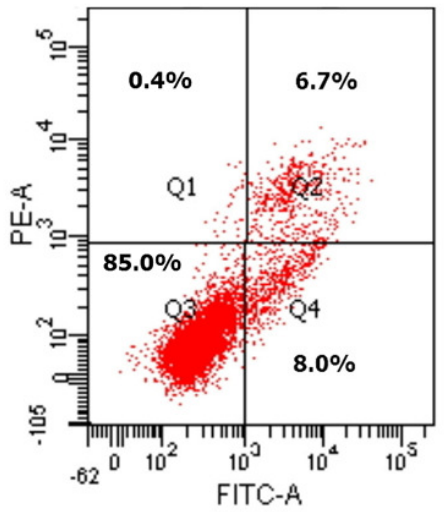

c.

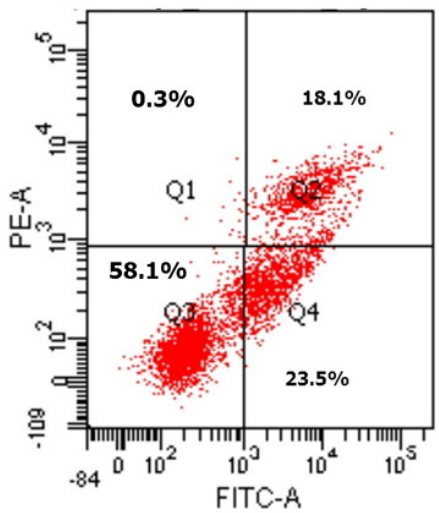

F.

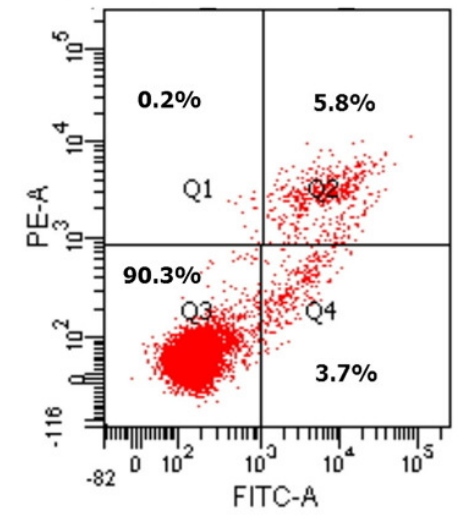

Figure $3 \mathrm{~A}$ and $3 \mathrm{D}$ showed the cell apoptosis analysis of $100 \mathrm{nM}-\mathrm{TR}$ and $100 \mathrm{nMN}-\mathrm{CR}$ of Hep-2 cell line after radiation

respectively; Figure 3B and 3E showed the cell apoptosis analysis of 100nM-TR and 100nMN-CR of M2e cell line after

cell line after radiation respectively. The vertical and horizontal axis in the Figure 3 standed for PI positive area and

FITC positive area respectively. Identified by flow cytometry, cells were divided into four sections: Q1: Annexin V-FITC

- PI +, was representative of mechanical error; Q2: Annexin V-FITC+ PI+, was representative of late apoptosis or 
271 early apoptosis of Hep-2 CSCs was 10.8\%; Figure 3E: early apoptosis of M2e CSCs was $8.0 \%$; Figure 3F: early 272 apoptosis of TU212 CSCs was 3.7\%; Figure 3D: late apoptosis of Hep-2 CSCs was 8.0\%; Figure 3E: late apoptosis of 273 M2e CSCs was 6.7\%; Figure 3F: late apoptosis of TU212 CSCs was 5.8\%) respectively after radiation.

274

275

276

277

278

279

280

281

282

283

284

285

286

287

288

289

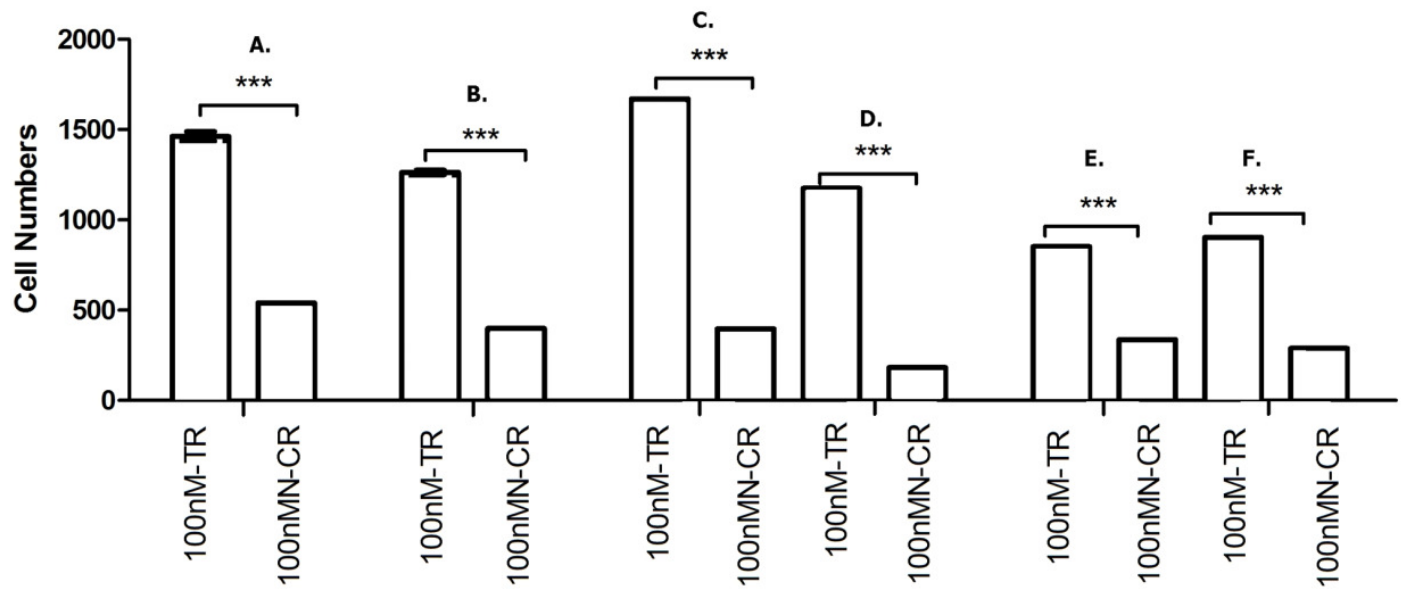

Figure 4. Overexpressed hsa-miR-138-2-3p induced cell apoptosis after radiation

Figure 4A and 4B showed the comparison of early and late apoptosis between 100nM-TR and 100nMN-CR of Hep-2 cell line after radiation respectively; Figure 4C and 4D showed the comparison of early and late apoptosis between 100nM-TR and 100nMN-CR of M2e cell line after radiation respectively; Figure 4E and 4F showed the comparison of early and late apoptosis between 100nM-TR and 100nMN-CR of TU212 cell line after radiation respectively. The vertical and horizontal axis in the Figure 4 standed for cell numbers and early/late apoptosis respectively. We found that the cell numbers of early apoptosis and late apoptosis of Hep-2, M2e, and TU212 CSCs induced by transfection of 100nM hsa-miR-138-2-3p were larger than that induced by transfection of $100 \mathrm{nM}$ nonsense oligonucleotides respectively after radiation, the differences between them were statistically significant $\left({ }^{* * *} P<0.001\right)$.

\subsection{Overexpressed hsa-miR-138-2-3p induced cell cycle arrest after radiation}

DNA content stained with PI were detected with flow cytometry, and cell cycle distribution of Hep-2, M2e, and TU212 cell lines were analyzed by ModFit software[17]. As Figure5 shown, the growth cycle of cell is divided into five stages, known as G0 phase, in which cell is quiescent, G1 and G2 phase, in which it increases in size, S phase, in which it duplicates its DNA, and M phase, in which it undergoes mitosis and divides. As shown in Figure 6 and Figure7, the percentage of Hep-2, M2e, 
and TU212 CSCs induced by transfection of 100nM hsa-miR-138-2-3p in G1 phase $(68.68 \%, 65.95 \%$,

$29165.24 \%)$ were more than that induced by transfection of $100 \mathrm{nM}$ nonsense oligonucleotides $(55.44 \%$,

$29256.90 \%, 59.23 \%$ ) respectively after radiation. While, the percentage of Hep-2, M2e, and TU212 CSCs

293 induced by transfection of $100 \mathrm{nM}$ hsa-miR-138-2-3p in S phase $(23.32 \%, 26.05 \%, 26.76 \%)$ were less

294 than that induced by transfection of $100 \mathrm{nM}$ nonsense oligonucleotides $(36.56 \%, 37.25 \%, 37.96 \%)$

295 respectively after radiation. The percentage of M2e, and TU212 CSCs induced by transfection of

296 100nM hsa-miR-138-2-3p in G2 phase $(8 \%, 8 \%)$ were more than that induced by transfection of

297 100nM nonsense oligonucleotides $(5.85 \%, 2.81 \%)$ respectively after radiation, but the percentage of

298 Hep-2 CSCs induced by transfection of 100nM hsa-miR-138-2-3p in G2 phase (8\%) was as the same

299 as that induced by transfection of $100 \mathrm{nM}$ nonsense oligonucleotides (8\%) after radiation. However, all

300 differences between them were not statistically significant.

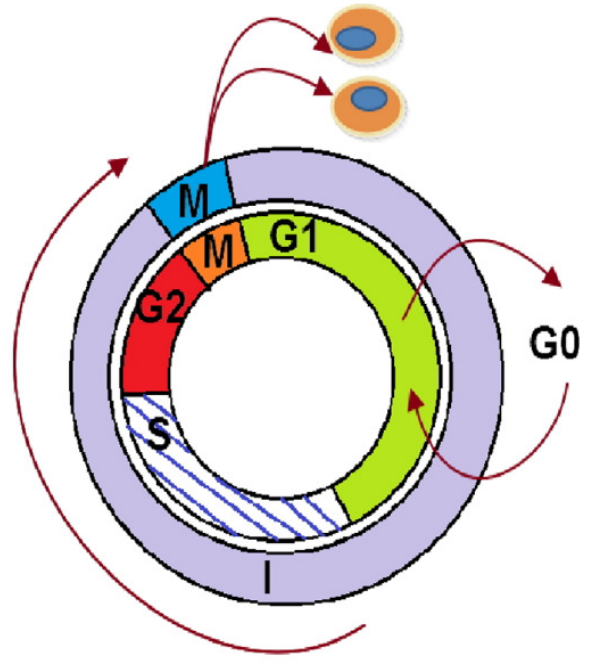

A.

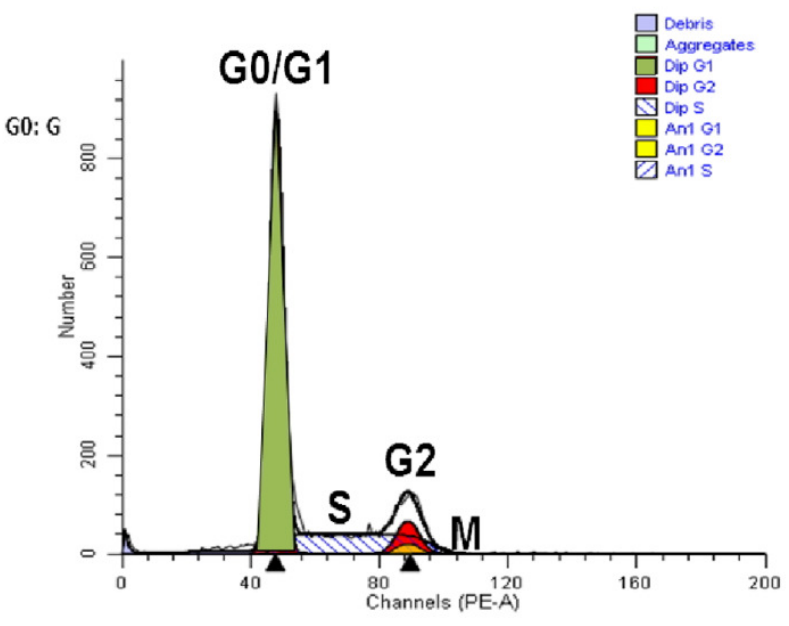

B.
301

302

303

304

305

306

307

Figure 5. Cell division cycle analysis

As shown in Figure 5A,5B, it is cell division cycle. $G 0$ is the abbreviation of Gap0, cell in $\mathrm{G} 0$ phase has left the cycle and has stopped dividing, and cell cycle starts with this phase; G1 is the abbreviation of Gap1, cell in G1 phase increase in size, and is ready for DNA synthesis; $S$ is the abbreviation of Synthesis, cell in $S$ phase is for DNA replication; $\mathrm{G} 2$ is the abbreviation of Gap2, cell in G2 phase continue growing; $M$ is the abbreviation of Mitosis, cell in M phase is stopped growing, and cellular energy is used for the orderly division into two daughter cells. 

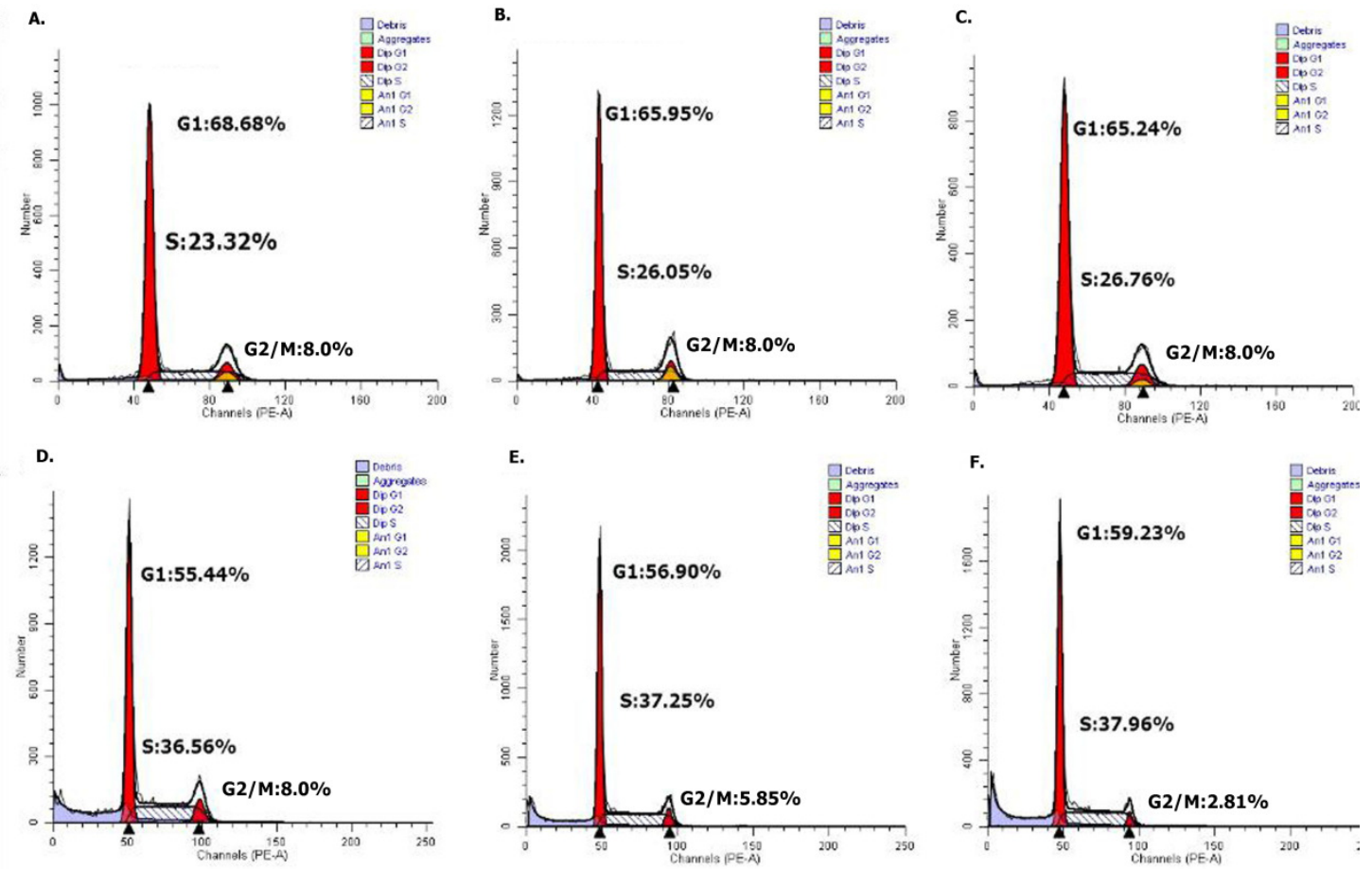

Figure 6. Overexpressed hsa-miR-138-2-3p arrested cell cycle at G1/S phase after radiation by flow cytometry

312 Figure $6 \mathrm{~A}$ and $6 \mathrm{D}$ showed the cell cycle analysis of 100nM-TR and 100nMN-CR of Hep-2 cell line after radiation

313 respectively; Figure $6 \mathrm{~B}$ and $6 \mathrm{E}$ showed the cell cycle analysis of 100nM-TR and 100nMN-CR of M2e cell line after

314 radiation respectively; Figure $6 \mathrm{C}$ and $6 \mathrm{~F}$ showed the cell cycle analysis of 100nM-TR and 100nMN-CR of TU212 cell

315 line after radiation respectively. The vertical and horizontal axis in the Figure 6 standed for cell numbers and cell

316 division cycle respectively. The percentage of Hep-2, M2e, and TU212 CSCs induced by transfection of 100nM hsa-

317 miR-138-2-3p in G1 phase $(68.68 \%, 65.95 \%, 65.24 \%)$ were more than that induced by transfection of $100 \mathrm{nM}$

318 nonsense oligonucleotides $(55.44 \%, 56.90 \%, 59.23 \%)$ respectively after radiation. While, the percentage of Hep-2,

$319 \mathrm{M} 2 \mathrm{e}$, and TU212 CSCs induced by transfection of $100 \mathrm{nM}$ hsa-miR-138-2-3p in S phase $(23.32 \%, 26.05 \%, 26.76 \%)$

320 were less than that induced by transfection of $100 \mathrm{nM}$ nonsense oligonucleotides $(36.56 \%, 37.25 \%, 37.96 \%)$

321 respectively after radiation. The percentage of M2e, and TU212 CSCs induced by transfection of 100nM hsa-miR-138-

$3222-3 p$ in G2 phase (8\%, 8\%) were higher than that induced by transfection of $100 \mathrm{nM}$ nonsense oligonucleotides $(5.85 \%$,

$3232.81 \%$ ) respectively after radiation, but the percentage of Hep-2 CSCs induced by transfection of 100nM hsa-miR-138- 
$3242-3 p$ in G2 phase (8\%) was as the same as that induced by transfection of 100nM nonsense oligonucleotides ( $8 \%$ )

325 after radiation.

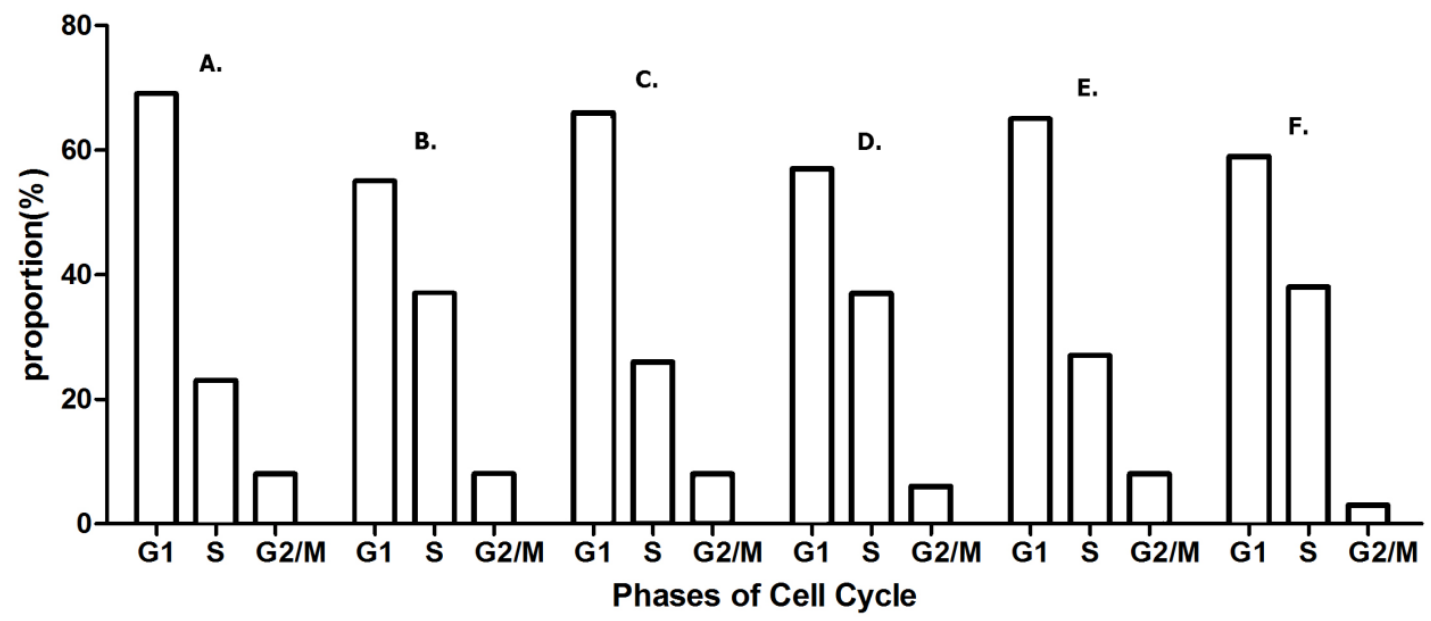

326

Figure 7. Overexpressed hsa-miR-138-2-3p arrested cell cycle at G1/S phase after radiation

328 Figure 7A and 7B showed the cell cycle analysis of 100nM-TR and 100nMN-CR of Hep-2 cell line after radiation respectively; Figure 7C and 7D showed the cell cycle analysis of 100nM-TR and 100nMN-CR of M2e cell line after radiation respectively; Figure 7E and 7F showed the cell cycle analysis of 100nM-TR and 100nMN-CR of TU212 cell line after radiation respectively. The vertical and horizontal axis in the Figure 7 standed for proportions and cell division phases respectively. The proportion of Hep-2, M2e, and TU212 CSCs induced by transfection of 100nM hsa-miR-1382-3p in G1 phase were higher than that induced by transfection of 100nM nonsense oligonucleotides respectively after radiation. While, the percentage of Hep-2, M2e, and TU212 CSCs induced by transfection of 100nM hsa-miR-138-2$3 p$ in $S$ phase were less than that induced by transfection of $100 \mathrm{nM}$ nonsense oligonucleotides respectively after radiation. The percentage of M2e, and TU212 CSCs induced by transfection of 100nM hsa-miR-138-2-3p in G2 phase were more than that induced by transfection of $100 \mathrm{nM}$ nonsense oligonucleotides respectively after radiation, but the percentage of Hep-2 CSCs induced by transfection of 100nM hsa-miR-138-2-3p in G2 phase was as the same as that induced by transfection of 100nM nonsense oligonucleotides after radiation. However, all differences between them were not statistically significant $(P>0.05)$.

341 2.4 Overexpressed hsa-miR-138-2-3p inhibited cell invasion after radiation

342 Cancer migration and invasion are serious and fatal steps in cancer progression [6,23], so it is 
343 important and urgent to identify therapeutic targets to prevent the metastases of cancer cells. We

344 found that hsa-miR-138-2-3p played a key role in inhibiting laryngeal CSCs invasion. Transwell

345 invasion assay demonstrated that the numbers of Hep-2, M2e, and TU212 CSCs that were

346 transfected into $50 \mathrm{nM}, 100 \mathrm{nM}$, and $150 \mathrm{nM}$ hsa-miR-138-2-3p penetrated through matrigel were lower

347 than that were transfected into $100 \mathrm{nM}$ nonsense oligonucleotides and PBS buffer at $24 \mathrm{~h}$ after

348 radiation, a significant difference between experimental teams and control teams (Figure 8,9 ). It was

349 important to note that the cell numbers of $100 \mathrm{nM}-\mathrm{TR}$ and $150 \mathrm{nM}-\mathrm{TR}$ were lower than $50 \mathrm{nM}-\mathrm{TR}$ and

350 the differences were statistically significant. However, this phenomenon was not investigated without

351 radiation, the cell numbers of $100 \mathrm{nM}-\mathrm{C}$ were not lower than $100 \mathrm{nMN}-\mathrm{C}$ and PBS-C (Figure 8,9 ).

352

353

354

355

356

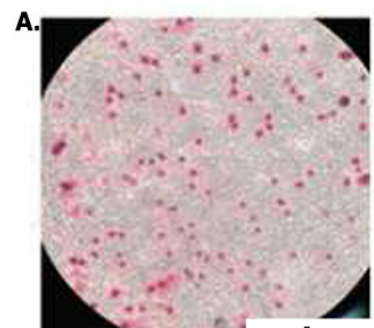

A.

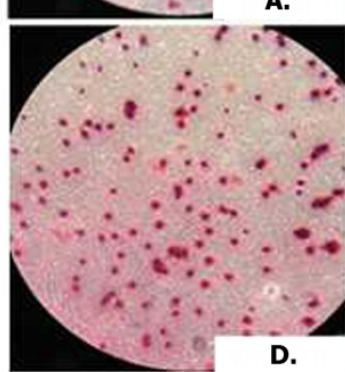

D.
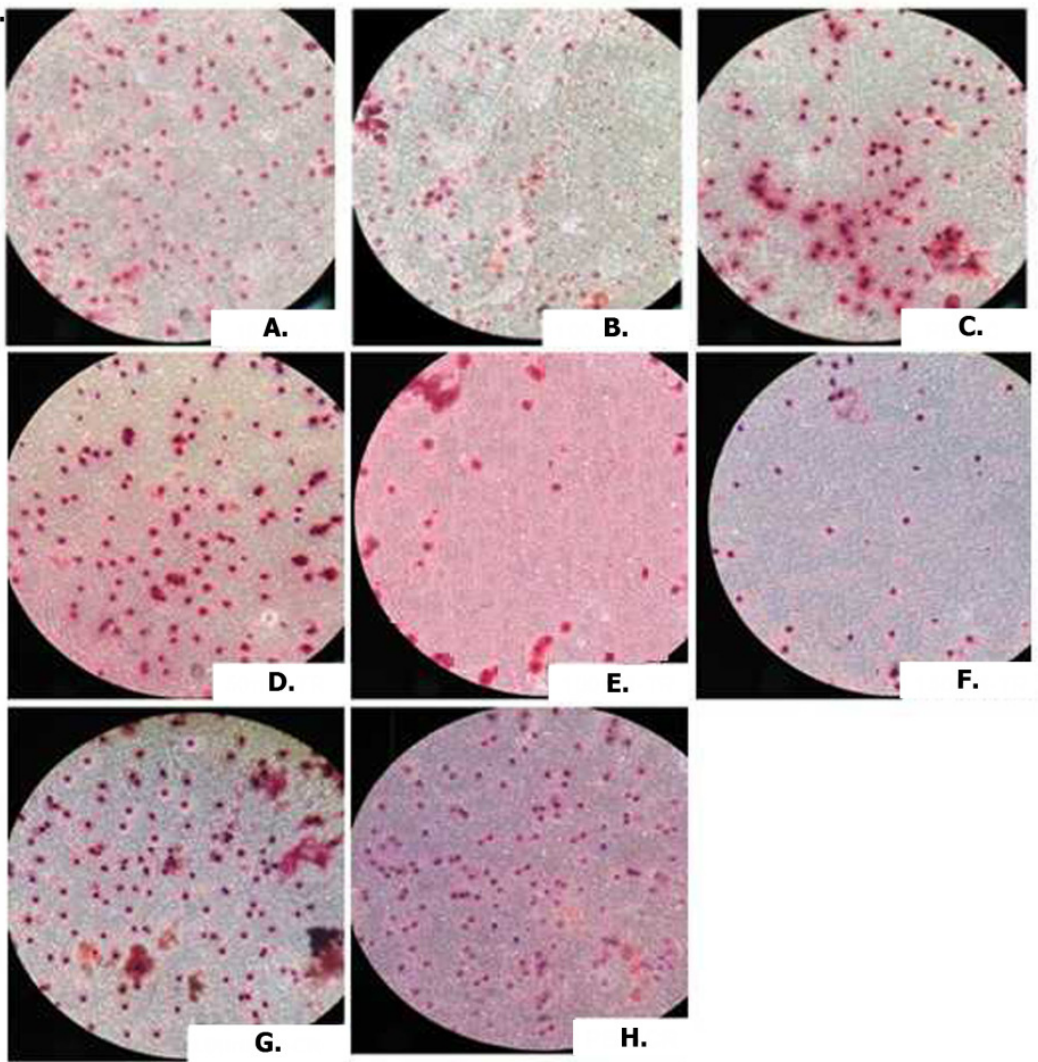

F.

Figure 8. Overexpressed hsa-miR-138-2-3p inhibited cell invasion by Transwell assay

Figure 8A, 8B and 8C showed Transwell Assay of 100nM-T, 100nMN-C and PBS-C of Hep-2 cell line without radiation respectively; Figure 8D, 8E, 8F, 8G and $8 \mathrm{H}$ showed Transwell Assay of 50nM-TR, 100nM-TR, 150nM-TR, 100nMNCR and PBS-CR of Hep-2 cell line 24h after radiation respectively. The cell numbers of experimental teams and 
357 control teams of Hep-2 CSCs were reduced at $24 \mathrm{~h}$ after radiation. Among them, Hep-2 CSCs were dropped most. The

A.

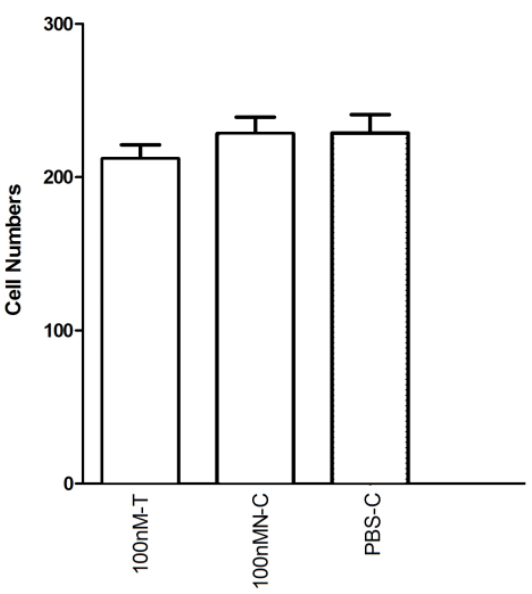

363

364

365

366

367

368

369

370

371

372

373

374

375

Figure 9. Overexpressed hsa-miR-138-2-3p inhibited laryngeal CSCs invasion

Figure 9A showed the comparison of Transwell Assay among 100nM-T, 100nMN-C and PBS-C of Hep-2 cell line without radiation respectively; Figure 9B showed the comparison of Transwell Assay of 50nM-TR, 100nM-TR, 150nM-

TR, 100nMN-CR and PBS-CR of Hep-2 cell line 24h after radiation respectively. The vertical and horizontal axis in the Figure 9A and 9B standed for cell numbers and treatments respectively. Transwell invasion assay demonstrated that the numbers of Hep-2 CSCs that were transfected into $50 \mathrm{nM}, 100 \mathrm{nM}$, and 150nM hsa-miR-138-2-3p penetrated through matrigel were lower than that were transfected into 100nM nonsense oligonucleotides and PBS buffer at 24h after radiation, a significant difference between experimental teams and control teams $\left({ }^{* * *} \mathrm{P}<0.001\right)($ Figure $9 \mathrm{~B})$. It was important to note that the cell numbers of $100 \mathrm{nM}-\mathrm{TR}$ and $150 \mathrm{nM}-\mathrm{TR}$ were lower than 50nM-TR and the differences were statistically significant $\left({ }^{* *} \mathrm{P}<0.001\right)$. However, this phenomenon was not investigated without radiation, the cell numbers of 100nM-C were not significantly lower than 100nMN-C and PBS-C (P>0.05) (Figure 9A).

\subsection{Overexpressed hsa-miR-138-2-3p reduced survival fraction after radiation}


376 100nM-TR, 100nM-CR, and non-transfection of Hep-2 cell line were treated with 0, 2, 4, 6, 8 Gy X-ray

377 irradiation respectively, and then were incubated for additional 14 days. After incubation, cells were

378 fixed with paraformaldehyde and the colonies (> 50 cells) were counted for each culture dish by a 379 light microscope. The survival fraction (SF) of 100nM-TR, 100nM-CR, and non-transfection of Hep-2 380 CSCs were evaluated. The SF of 100nM-TR of all laryngeal CSCs were lower than that of 100nMN$381 \mathrm{CR}$ and non-transfection, and the difference between 100nM-TR and non-transfection were 382 statistically significant.

383

384

385

386

387

388

389

390

391

392

393

394

395

396

397

398
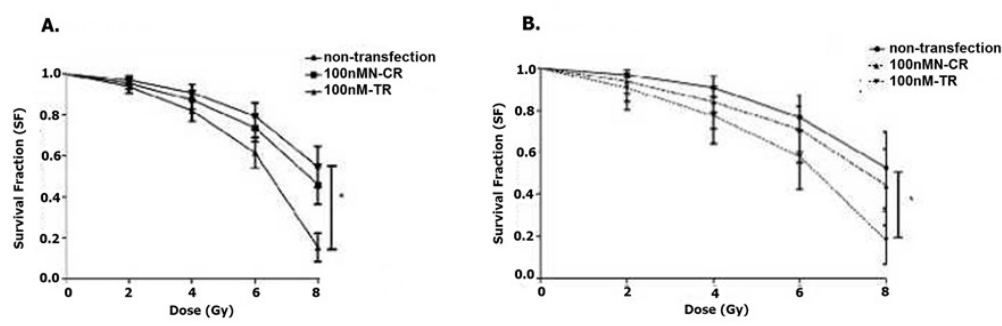

Figure 10 Overexpressed hsa-miR-138-2-3p reduced survival fraction after radiation

Figure 10A, 10B and 10C showed the comparison of survival analysis among $100 \mathrm{nM}-\mathrm{TR}, 100 \mathrm{nMN}-\mathrm{CR}$ and nontransfection of Hep-2, M2e, and TU212 cell lines after radiation respectively. The vertical and horizontal axis in the Figure 10 standed for survival fraction and does(Gy) respectively. 100nM-TR, 100nM-CR, and non-transfection of Hep-2, M2e and TU212 were treated with 0, 2, 4, 6, 8 Gy X-ray irradiation respectively, and the survival fraction of 100nM-TR of all laryngeal CSCs were lower than that of $100 \mathrm{nMN}-\mathrm{CR}$ and non-transfection, and the difference between 100nM-TR and non-transfection were statistically significant ( $\left.{ }^{*} P<0.05\right)$.

\subsection{Overexpressed hsa-miR-138-2-3p promoted DNA damage after radiation}

The degree of DNA damage after radiation was measured by comet assay. As shown in Figure 11, the appearance of "comet" with fragmented DNA (tail) being separated from undamaged nuclear DNA (head) was seen in 100nM-TR and 100nMN-CR of Hep-2, M2e, and TU212 CSCs after radiation. We found that the "heads" of "comet" of 100nM-TR were smaller than that of $100 \mathrm{nMN}-\mathrm{CR}$, while the "tails" of "comet" of 100nM-TR were longer than that of 100nMN-CR. Further, the measurements were made by Comet Assay IV software to calculate the tail movement (\%). As Figure 12 shown, the tail movement of $100 \mathrm{nM}-\mathrm{TR}$ of all laryngeal CSCs were higher than that of $100 \mathrm{nMN}$ - 
399 CR, the differences between 100nM-TR and 100nMN-CR of Hep-2 cells were statistically significant.

400 These data were indicated that the DNA damage of $100 \mathrm{nM}-\mathrm{TR}$ were more serious than that of 401 100nM-CR in laryngeal CSCs after radiation.

A.

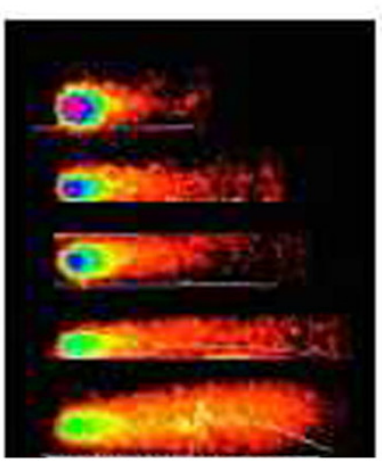

D.

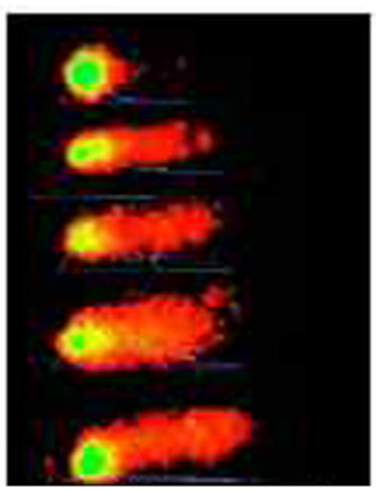

B.

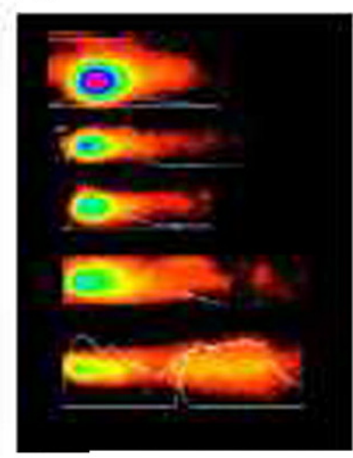

E.

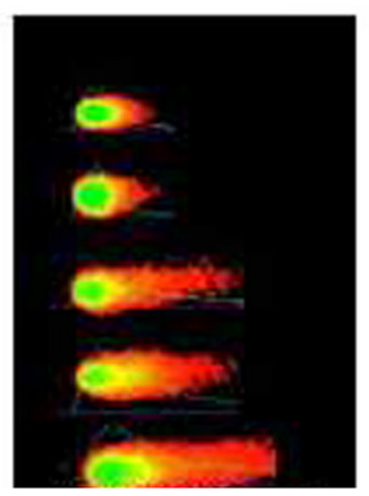

C.

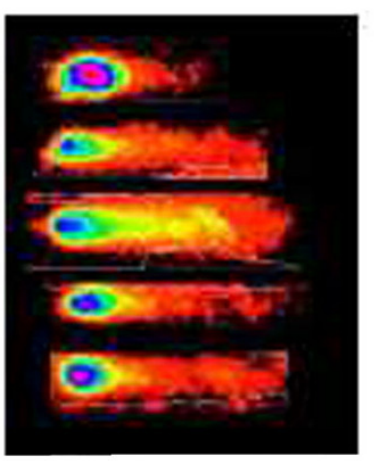

F.

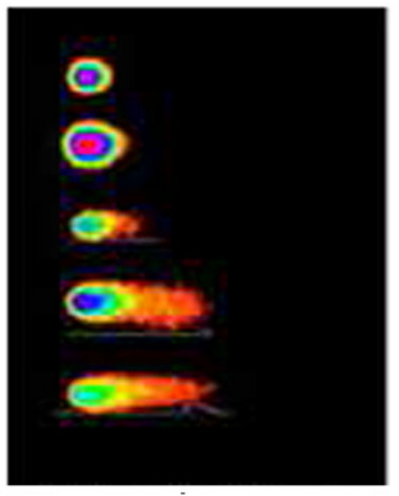

402

403

404

405

406

407

408

409

410

411

Figure 11 Overexpressed hsa-miR-138-2-3p promoted DNA damage after radiation by Comet assay

Figure 11A and 11D showed the DNA damage analysis of $100 \mathrm{nM}-\mathrm{TR}$ and $100 \mathrm{nMN}-\mathrm{CR}$ of Hep-2 cell line after radiation respectively; Figure 11B and 11E showed the DNA damage analysis of 100nM-TR and 100nMN-CR of M2e cell line after radiation respectively; Figure $11 \mathrm{C}$ and $11 \mathrm{~F}$ showed the DNA damage analysis of $100 \mathrm{nM}$-TR and 100nMN-CR of TU212 cell line after radiation respectively. As shown in Figure 11, the appearance of "comet" with fragmented DNA (tail) being separated from undamaged nuclear DNA (head) was seen in 100nM-TR and 100nMN-CR of Hep-2, M2e, and TU212 CSCs after radiation. It was found that the "heads" of "comet" of 100nM-TR were smaller than that of $100 \mathrm{nMN}-\mathrm{CR}$, while the "tails" of "comet" of $100 \mathrm{nM}-\mathrm{TR}$ were longer than that $100 \mathrm{nMN}-\mathrm{CR}$. These data 
412 were indicated that the DNA damage of 100nM-TR were more serious than that of 100nM-CR in laryngeal CSCs after 413 radiation.

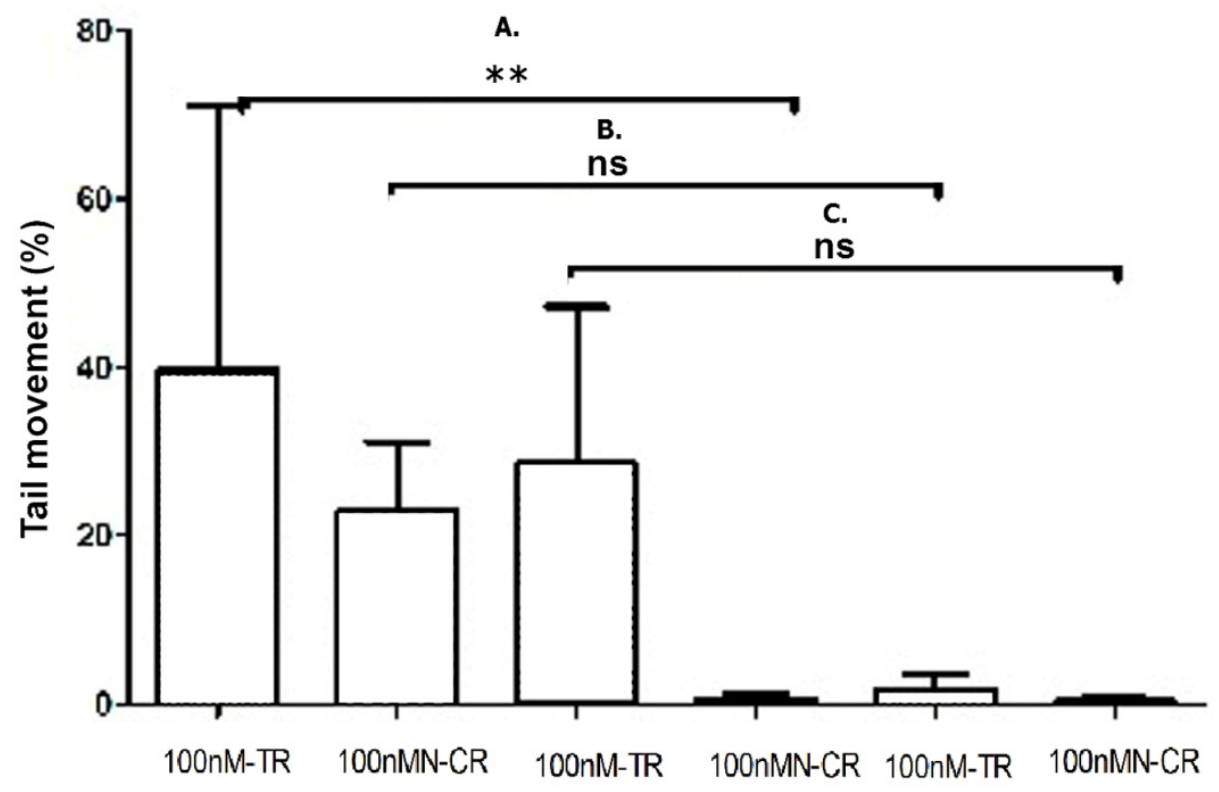

Figure 12 . Overexpressed hsa-miR-138-2-3p promoted DNA damage after radiation

416 Figure 12A showed the comparison of DNA damage between 100nM-TR and 100nMN-CR of Hep-2 cell line after

417 radiation respectively; Figure 12B showed the comparison of DNA damage between 100nM-TR and 100nMN-CR of

418 M2e cell line after radiation respectively; Figure 12C showed the comparison of DNA damage between 100nM-TR and

419 100nMN-CR of TU212 cell line after radiation respectively. The vertical and horizontal axis in the Figure 12 standed for

420 Tail movement (\%) and treaments respectively. As Figure 12 shown, the tail movement of 100nM-TR of all laryngeal

421 cell lines were higher than that of 100nMN-CR, and the differences between 100nM-TR and 100nMN-CR of Hep-2

422 CSCs were statistically significant $\left({ }^{* *} \mathrm{P}<0.01\right)$. These data were indicated that the DNA damage of $100 \mathrm{nM}-\mathrm{TR}$ were

423 more serious than that of $100 \mathrm{nM}-\mathrm{CR}$ in laryngeal CSCs after radiation.

424 3. Overexpressed hsa-miR-138-2-3p regulated signal transduction pathway of laryngeal CSCs

425 after radiation

426 3.1 Overexpressed hsa-miR-138-2-3p inhibited Wnt/ $\beta$-catenin pathway

427 It was known that accumulation of $\beta$-catenin leaded to abnormal activation of Wnt/ $\beta$-catenin signaling

428 pathway and reinforce radiation resistance. As shown in Figure 13-14, the expression of $\beta$-catenin in 
$429100 \mathrm{nM}-\mathrm{TR}$ of all laryngeal CSCs were reduced more than that in $100 \mathrm{nMN}-\mathrm{CR}$, and the results 430 between $100 \mathrm{nM}-\mathrm{TR}$ and $100 \mathrm{nMN}-\mathrm{CR}$ of all laryngeal CSCs were significant difference. It suggested 431 that overexpression of hsa-miR-138-2-3p reduced the expression of $\beta$-catenin, further inhibited the 432 activity of $\mathrm{Wnt} / \beta$-catenin pathway and reduced the resistance of the laryngeal CSCs to radiation.

A.

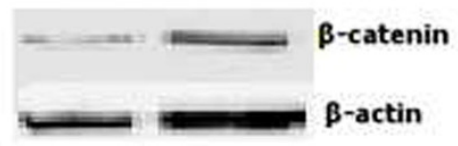

B.

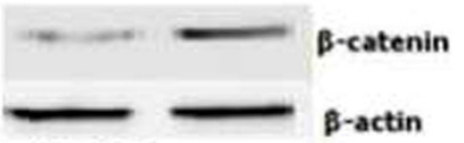

c.

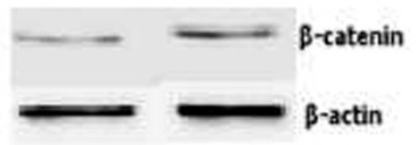

433

Figure 13. Overexpressed hsa-miR-138-2-3p down-regulated expression of $\beta$-catenin As shown in Figure 13, the expression of $\beta$-catenin in 100nM-TR of Hep-2 (A), M2e (B) and TU212 (C) CSCs were reduced more than that in $100 \mathrm{nMN}-\mathrm{CR}$.

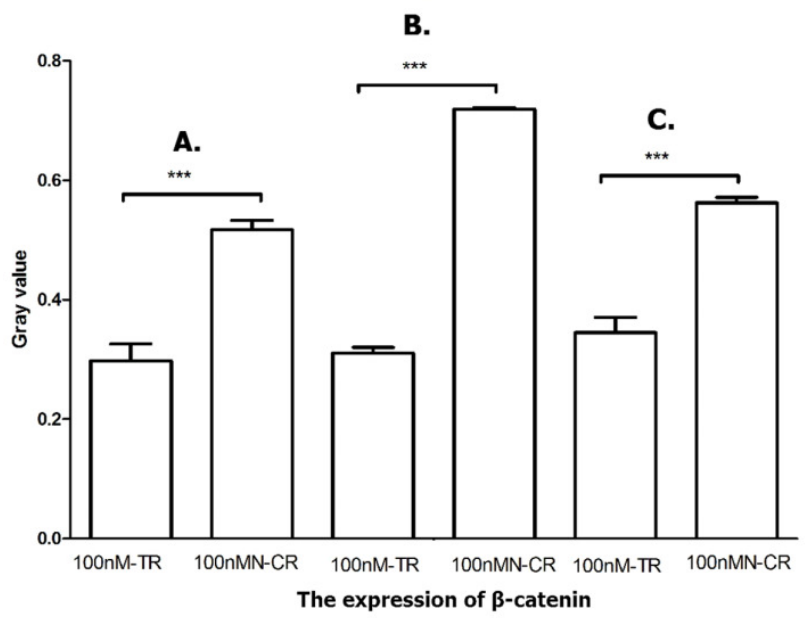

Figure 14. Overexpressed hsa-miR-138-2-3p inhibited Wnt/ $\beta$-catenin pathway

As Figure 14 shown, the vertical and horizontal axis standed for Gary value and treaments respectively. It suggested that the expression of $\beta$-catenin between 100nM-TR and 100nMN-CR of Hep-2 (A), M2e (B) and TU212 (C) CSCs were significant difference $\left.{ }^{* * *} \mathrm{P}<0.001\right)$.

\subsection{Overexpressed hsa-miR-138-2-3p inhibited Hippo/YAP1 pathway}

Hippo signal pathway regulated cell prolifertion, development and progression. Ancillary transcription factor YAP1 played a key role in Hippo pathway. Knocking out nuclear YAP1 can inhibit tumor cell proliferation, while increased expression of YAP1 can promote cell growth and migration and inhibit apoptosis. As shown in Figure 15-16, the expression of YAP1 in 100nM-TR of all laryngeal CSCs 
447 were down-regulated much more than that in $100 \mathrm{nMN}-\mathrm{CR}$, and the results between 100nM-TR and $448100 \mathrm{nMN}-\mathrm{CR}$ of all laryngeal CSCs were significant difference. It suggested that overexpression of 449 hsa-miR-138-2-3p reduced the expression of YAP1, further controlling Hippo signal pathway to 450 weaken radio-resistance of laryngeal CSCs.

A.

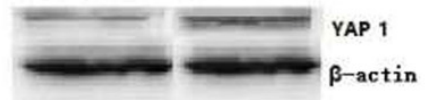

B.

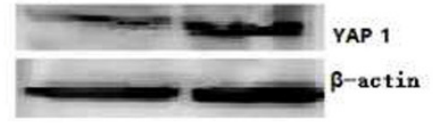

C.

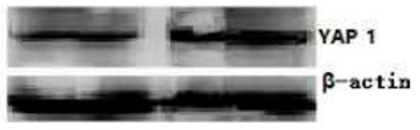

451

Figure 15. Overexpressed hsa-miR-138-2-3p down-regulated expression of YAP1

As shown in Figure 15, the expression of YAP1 in 100nM-TR of Hep-2 (A), M2e (B) and TU212 (C) CSCs were

454 reduced more than that in $100 \mathrm{nMN}-\mathrm{CR}$.

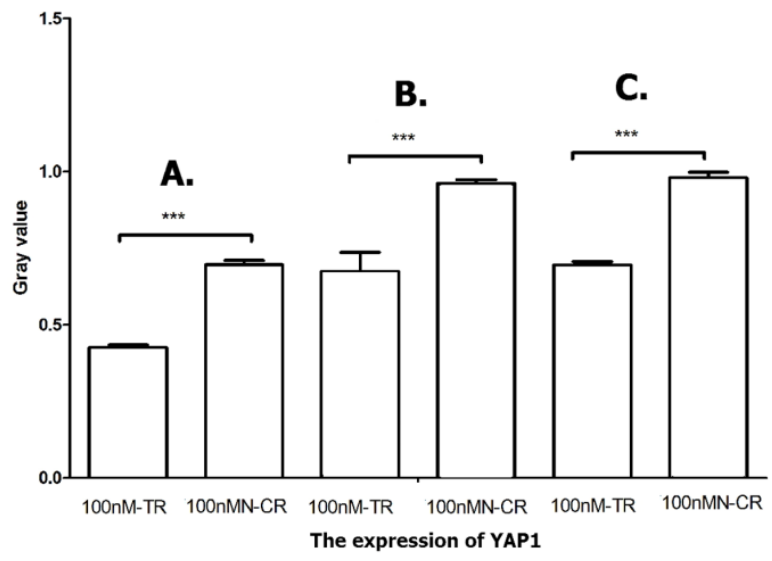

455

456

Figure 16. Overexpressed hsa-miR-138-2-3p inhibited Hippo/YAP1 pathway

457

As Figure 16 shown, the vertical and horizontal axis standed for Gary value and treaments respectively. It suggested

that the expression of YAP1 between 100nM-TR and 100nMN-CR of Hep-2 (A), M2e (B) and TU212 (C) CSCs were

459 significant difference $\left.{ }^{* * *} \mathrm{P}<0.001\right)$.

\subsection{Overexpressed hsa-miR-138-2-3p activated JNK1/p38/MAPK pathway}

461 MAPK/JNK signal pathway was activated by radiation, and enhanced the radio-sensitivity of tumor

462 cells by its association with radiation-induced DNA damage [16,18]. As shown in Figure 17-19, the

463 expression of JNK1 and p38 in 100nM-TR of all laryngeal CSCs were improved much more than that

464 in $100 \mathrm{nMN}-\mathrm{CR}$, and the results between 100nM-TR and 100nMN-CR of all laryngeal CSCs were 
465 significant difference. It suggested that overexpression of hsa-miR-138-2-3p promoted the 466 expression of JNK1 and p38, further activating JNK1/p38/MAPK signal pathway to increase radio467 sensitivity of laryngeal CSCs.

468

469

472

473

474

475

476

477
A.

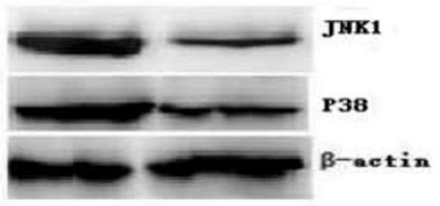

B.

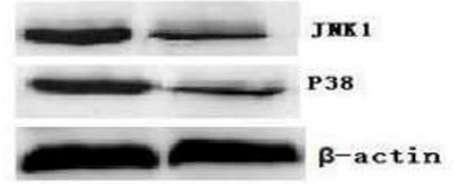

c.

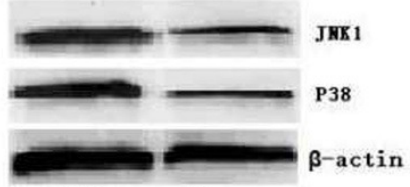

Figure 17. Overexpressed hsa-miR-138-2-3p up-regulated expression of JNK1 and p38

As shown in Figure 17, the expression of JNK1 and p38 in 100nM-TR of Hep-2 (A), M2e (B) and TU212 (C) CSCs were improved more than that in $100 \mathrm{nMN}-\mathrm{CR}$.

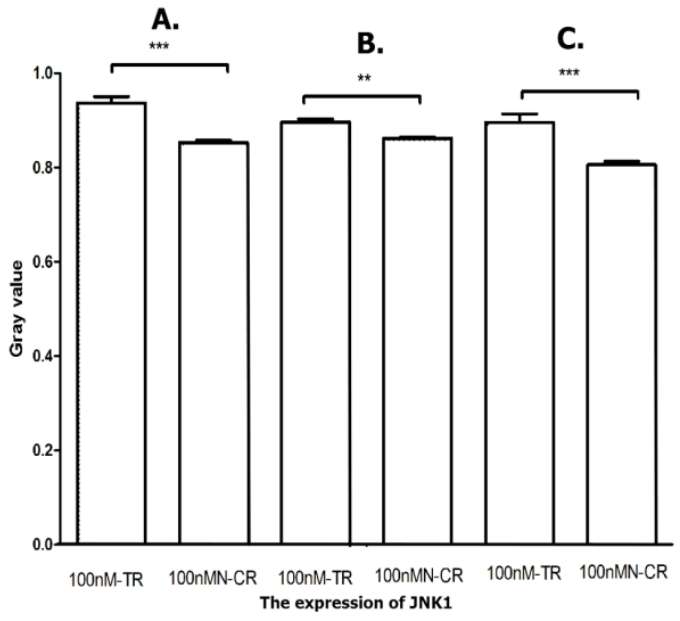

Figure 18. Overexpressed hsa-miR-138-2-3p activated JNK1/MAPK pathway

As Figure 18 shown, the vertical and horizontal axis standed for Gary value and treaments respectively. It suggested that the expression of JNK1 between 100nM-TR and 100nMN-CR of Hep-2 (A), M2e (B) and TU212 (C) CSCs were significant difference $\left({ }^{* *} \mathrm{P}<0.01,{ }^{* * *} \mathrm{P}<0.001\right)$. 


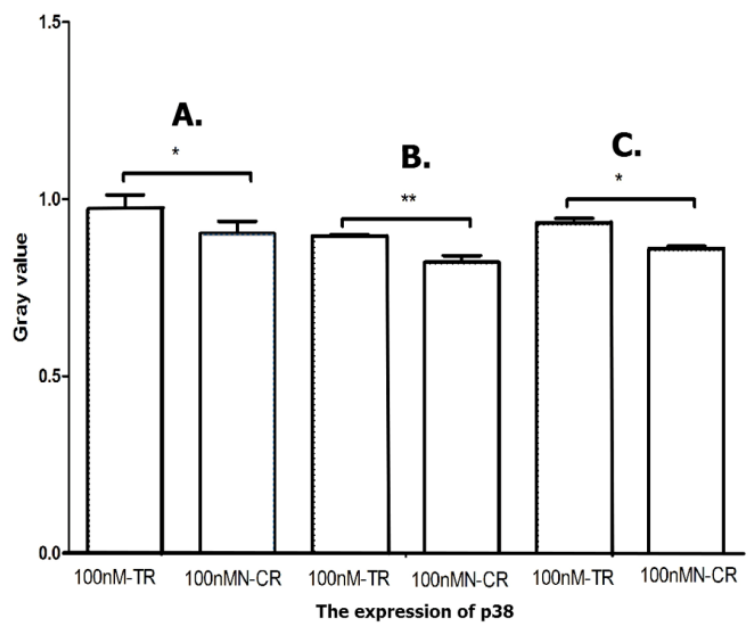

478

479

480

481

482

483

484

485

486

487

488

489

490

491

492

493

494

495

496

Figure 19. Overexpressed hsa-miR-138-2-3p activated p38/MAPK pathway

As Figure 19 shown, the vertical and horizontal axis standed for Gary value and treaments respectively. It suggested that the expression of p38 between 100nM-TR and 100nMN-CR of Hep-2 (A), M2e (B) and TU212 (C) CSCs were significant difference $\left({ }^{*} \mathrm{P}<0.05,{ }^{* *} \mathrm{P}<0.01\right)$.

\section{Discussion}

In recent years, a large number of studies have focused on miRNA and cancer radiosensitivity. miRNAs played important roles in cell proliferation, invasion, apoptosis and cell cycle arrest, and aggressive growth, development, and metastasis are another characteristics of radioresistance[16,23]. Balca-Silva et al[19]found that over-expressed miR-34 enhanced the radiosensitivity of non-small cell lung cancers. In gastric cancer, knocking out miR-221 and miR-222 could inhibit proliferation and invasion, increase radiosensitivity of gastric carcinoma cells[20]. So far, however, there is no report on the relationship between hsa-miR-138-2-3p and radiation sensitivity of laryngeal cancer. In our present study, overexpressed hsa-miR-138-2-3p played an key role in decreasing laryngeal CSCs proliferation and invasion; increasing the proportion of early and late apoptosis in laryngeal CSCs; raising G1 phase arrest; and down-regulating the proportion of S stage cells of cell cycle that were related to radio-resistance in laryngeal CSCs.

Wnt/ $\beta$-catenin signal transduction pathway regulated many cellular processes such as cell proliferation, apoptosis and aggressiveness, of which, $\beta$-catenin is the major factor. Che et al [23] 
497 investigated that Cox-2 inhibitor NS398 inhibited the expression of DNA-PKCs and controlled Wnt/ß-

498 catenin pathway to improve the radiation sensitivity of Eca109 cells. Chang et al[24] suggested that

499 the radiation resistance of AMC-HN-9 cells were decreased remarkably when $\beta$-catenin was knocked

500 out. Wang's study[25] showed that the excessive expression of IncRNA- p21 improve the

501 radiosensitivity of cells by inhibiting $W n t / \beta$-catenin signaling pathway. Regulating upstream signaling

502 molecules of $\mathrm{Wnt} / \beta$-catenin pathway and promoting degradiation of $\beta$-catenin may become new

503 anticancer treatments [26-28]. Recent studies have revealed that Hippo signaling pathway played a

504 role in the growth of many types of cancer cells, and YAP1 was associated with lung cancer and

505 ovarian malignant tumors. Cell proliferation was inhibited by knocking out YAP1, while increased

506 expression of YAP1 accelerated cell growth and migration. YAP1 was also called oncoprotein. MAPK

507 signal pathway plays a role in regulating many cellular activities, such as growth, differentiation and

508 stress reaction. Many signal transduction pathways related to radiation were regulated by MAPK

509 family, including extracellular signal regulating kinase (ERK), c-Jun amino end kinase (JNK) and p38

510 MAPK pathway. JNK1 and JNK2, the stress inducing protein kinases, induce the phosphorylation of

511 transcription factor c-Jun. MAPK pathway activated in this way is also called stress activating protein

512 kinase (SAPK) pathway. Studies have shown that apoptosis initiation and cell cycle arrest were

513 closely related to MAPK/p38 and MAPK/JNK1 pathway. Mediating MAPK/p38 and MAPK/JNK1

514 pathways can affect the radio-sensitivity of tumor cells. MAPK signaling pathway is considered to

515 have an important effect on the radiation sensitivity of tumor cells for its association with radiation-

516 induced DNA damage [16]. The activation of JNK and associated signaling pathway is related to

517 apoptosis. Activation of SAPK/JNK leads to cell radio-sensitivity, and vice versa [18]. Wang et al[28]

518 and Bulavin et al[29] found that MAPK/p38 signaling pathway regulated the transition of G2 / M phase

519 in mammalian cells and participated in G2 arrest. Wakita et al[30]showed that Reg la-expressing cells

520 activated MAPK/JNK1 pathway to increase JNK1 protein expression so as to improve the radio-

521 sensitivity of esophageal squamous cell carcinomas. From the present study, we investigated that

522 overexpression of hsa-miR-138-2-3p reduced the expressions of $\beta$-catenin and YAP1 in the laryngeal 
523 CSCs after radiation, further Wnt/ $\beta$-catenin and Hippo/YAP1 signal pathways were inhibited to

524 weaken radio-resistance of laryngeal CSCs.

525

526 Conclusion

527 The present research indicated that overexpressed hsa-miR-138-2-3p played an key role in

528 decreasing laryngeal CSCs proliferation and invasion; increasing the proportion of early and late

529 apoptosis in laryngeal CSCs; raising G1 phase arrest; and down-regulating the proportion of S stage

530 cells of cell cycle that were related to radio-resistance in laryngeal CSCs. Overexpressed hsa-miR-

531 138-2-3p regulated signal transduction pathway of laryngeal CSCs after radiation. Over-expression of

532 hsa-miR-138-2-3p down-regulated the expression of $\beta$-catenin and YAP1 in the laryngeal CSCs after

533 radiation, further Wnt/ $\beta$-catenin and Hippo/YAP1 signal pathways were inhibited to weaken radio-

534 resistance of laryngeal CSCs. While the expressions of JNK1 and p38 were promoted,

$535 \mathrm{JNK} 1 / \mathrm{p} 38 / \mathrm{MAPK}$ signal pathway was activated to increase radio-sensitivity of laryngeal CSCs. These

536 results are useful for a better understanding of hsa-miR-138-2-3p in laryngeal CSCs, and prove hsa-

537 miR-138-2-3p as a promising biomarker and target for diagnosis and novel anti-cancer therapy for

538 laryngeal cancers.

539 References

540 [1]Kiera Rycaj, Dean G. Tang. (2014)Cancer stem cells and radioresistance [J]. Int J Radiat Biol. 541 90(8): 615-621.

542 [2] Claudia Peitzsch, Ina Kurth, Leoni Kunz-Schughart, Michael Baumann, Anna Dubrovska.

543 (2013)Discovery of the cancer stem cell related determinants of radioresistanc e[J]. Radiother Oncol.

544108 (2013): 378-387.

545 [3]Selcuk Colak, Jan Paul Medema.(2014)Cancer stem cells-important players in tumor therapy

546 Resistance [J]. FEBS Journal. 281,4779-4791.

547 [4] Kobayashi Y, Sugiura T, Shimoda M, Ishii K, Akimoto N, Yoshihama N, Mori Y. (2014)Knockdown

548 of the T-box transcription factor Brachyury increases sensitivity of adenoid cystic carcinoma cells to 
549 chemotherapy and radiation in vitro: Implications for a new therapeutic principle [J]. Int J Oncol. 44:

$550 \quad 1107-1117$.

551 [5] Wu J, Lai G, Wan F, Xiao Z, Zeng L, Wang X, Ye F, Lei T. (2012)Knockdown of checkpoint kinase

5521 is associated with the increased radiosensitivity of glioblastoma stem-like cells [J]. Tohoku J Exp 553 Med. 226(4): 267-274.

554 [6]Lu-Qing Zhao, Ann M. Bode, Ya Cao, Zi-Gang Dong. (2012)Regulatory mechanisms and clinical

555 perspectives of miRNA in tumor radiosensitivity [J]. Carcinogenesis. 33(11): 2220-2227.

556 [7]Huang CX, Zhu Y, Duan GL, Yao JF, Li ZY, Li D, Wang QQ. (2013)Screening for MiRNAs Related

557 to Laryngeal Squamous Carcinoma Stem Cell Radiation [J]. Asian Pac J Cancer Prev. 14(8): 45335584537.

559 [8]Heike Immervoll, Dag Hoem, Per Øystein Sakariassen, Ole Johnny Steffensen, Anders Molven. 560 (2008) Expression of the "stem cell marker" CD133 in pancreas and pancreatic ductal 561 adenocarcinomas [J]. BMC Cancer. 8: 1-14.

562 [9] Piero Dalerba, Scott J. Dylla, In-Kyung Park, Rui Liu, Xinhao Wang, Robert W. Cho, Timothy Hoey, 563 Austin Gurney, Emina H. Huang, Diane M. Simeone, Andrew A. Shelton, Giorgio Parmiani, Chiara 564 Castelli, Michael F. Clarke. (2007)Phenotypic characterization of human colorectal cancer stem cells 565 [J]. PNAS. 104: $10158-10163$.

566 [10]Benjamin P Lewis, Christopher B Burge, David P Bartel. (2005)Conserved Seed Pairing, Often

567 Flanked by Adenosines, Indicates that Thousands of Human Genes are MicroRNA Targets [J]. Cell. 568 120: 15-20.

569 [11]Doron Betel, Manda Wilson, Aaron Gabow, Debora S. Marks, Chris Sander. (2008) MicroRNA 570 target predictions with expression profiles. The microRNA.org resource: targets and expression [J]. $571 \quad$ Nucleic Acids Res. 36: D149-D153.

572 [12]Ana Kozomara, Sam Griffiths-Jones. (2014) miRBase: annotating high confidence microRNA 573 using deep sequencing data [J]. Nucleic Acids Res. 42: D68-D73.

574 [13]Jun-Hao Li, Shun Liu, Hui Zhou, Liang-Hu Qu, Jian-Hua Yang. (2014) starBase v2.0: decoding 
575 miRNA-ceRNA, miRNA-ncRNA and protein-RNA interaction networks from large-scale CLIP-Seq 576 data [J]. Nucleic Acids Res. 42: D92-D97.

577 [14]Na Huang, Jing Lin, Jian Ruan, Ning Su, Ruzhai Qing, Feiye Liu, Benfu He, Chengwei Lv, 578 Dayong Zheng, Rongcheng Luo. (2012)MiR-219-5p inhibits hepatocellular carcinoma cell 579 proliferation by targeting glypican-3 [J]. FEBS Lett. 586: 884-891.

580 [15]Xiao Wang, Jian Wu, Yiwei Lin, Yi Zhu, Xianglai Xu, Xin Xu, Zhen Liang, Shiqi Li, Zhenghui Hu,

581 Xiangyi Zheng, Liping Xie. (2014)microRNA-320c inhibits tumorous behaviors of bladder cancer by

582 targeting Cyclin-dependent kinase 6 [J]. J Exp Clin Cancer Res. 33: 69-80.

583 [16]Chann Lagadec, Erina Vlashi, Lorenza Della Donna, Carmen Dekmezian, Frank Pajonk.

584 (2012)Radiation-induced reprogramming of breast cancer cells [J]. Stem Cells. 30: 833-844.

585 [17]Shunsuke Nojiri, Takashi Joh. (2014)Albumin Suppresses Human Hepatocellular Carcinoma 586 Proliferation and the Cell Cycle [J]. Int J Mol Sc. 15(3): 5163-5174.

587 [18]Chanatip Methee trairut, Frank J. Slack. (2013) MicroRNAs in the lonizing Radiation Response 588 and in Radiotherapy [J]. Curr Opin Genet Dev. 23: 12-19.

589 [19] JOANA BALÇA-SILVA, SÍLVIA SOUSA NEVES, ANA CRISTINA GONÇALVES, ANA 590 MARGARIDA ABRANTES, JOÃO CASALTA-LOPES, MARIA FILOMENA BOTELHO, ANA 591 BELA SARMENTO-RIBEIRO, HENRIQUETA COIMBRA SILVA . (2012)Effect of miR-34b

592 overexpression on the radiosensitivity of non-small cell lung cancer cell lines [J]. Anticancer Res. 593 32(5): 1603-1609.

594 [20]Zhang Chun-zhi, Han Lei, Zhang An-ling, Fu Yan-chao, Yue Xiao, Wang Guang-xiu, Jia Zhi-fan, 595 Pu Pei-yu, Zhang Qing-yu, Kang Chun-sheng. (2010)MicroRNA-221 and micro-RNA-222 regulate 596 gastric carcinoma cell proliferation and radioresistance by ta targeting TPEN [J]. BMC Cancer. 10(10): 597367.

598 [21]Ping Jiang, En-Y Rao, Na Meng, Yong Zhao, Jun-J Wang. (2015) MicroRNA-17-92signifieantly 599 enhances radioresistance in human mantle cell lymphoma cells [J]. Radiat Oncol, 5(5):100.

600 [22]Yong Wu, Guo-Long Liu, Si-Hong Liu, Cai-Xia Wang, Yan-Li Xu, Yi Ying, Ping Mao. 
601 (2012)MicroRNA-148b enhances the Radiosensitivity of non-Hodgkin's LymPhoma cells by 602 promoting radiation-induced apoptosis [J]. J Radiat Res. 53(4): 516-525.

603 [23]Kumari L. Andarawewa, Anna C. Erickson, William S. Chou, Sylvain V. Costes, Philippe Gascard, 604 Joni D. Mott, Mina J. Bissell, Mary Helen Barcellos-Hoff. (2007)lonizing radiation predisposes 605 nonmalignant human mammary epithelial cells to undergo transforming growth factor beta induced 606 epithelial to mesenchymal transition [J]. Cancer Res. 67: 8662-8670.

607 [24]Chang HW, Nam HY, Kim HJ, Moon SY, Kim MR, Lee M, Kim G, Kim SW, Kim SY. (2016) Effect 608 of $\beta$-catenin silencing in overcoming radioresistance of head and neck cancer cells by antagonizing 609 the effects of AMPK on Ku70/Ku80 [J]. Head Neck. Dec 29. doi: 10.1002/hed.24347.

610 [25] Guangyu Wang, Zhiwei LiQi Zhao, Yuanyuan Zhu, Ci Zhao, Xin Li, Zhigang Ma, Xiaobo Li, 611 Yanqiao Zhang. (2014) LincRNA-p21 enhances the sensitivity of radiotherapy for human colorectal 612 cancer by targeting the Wnt/ß-catenin signaling pathway [J]. Oncol Rep. 31(4): 1839-1845.

613 [26] Na Huang, Jing Lin, Jian Ruan, Ning Su, Ruzhai Qing, Feiye Liu, Benfu He, Chengwei Lv,

614 Dayong Zheng, Rongcheng Luo (2012) MiR-219-5p inhibits hepatocellular carcinoma cell

615 proliferation by targeting glypican-3 [J]. FEBS Lett. 586: 884-891.

616 [27]Lin Qi, Ping Wu, Xin Zhang, Yuan-Zheng Qiu, et al. (2012) Inhibiting ERp29 expression

617 enhances radiosensitivity in human nasopharyngeal carcinoma cell lines [J]. Med Oncol. 29: 721-728.

618 [28]Qi Wang, Hui-Huan Tang, Shan-Shan Yin, Chao Dong. (2013)Downregulation of microRNA-138

619 enhances the proliferation, migration and invasion of cholangiocarcinoma cells through the 620 upregulation of RhoC/p-ERK/MMP-2/MMP-9 [J]. Oncol Rep. 29: 2046-2052.

621 [29]Kobayashi E, Satow R, Ono M, Masuda M, Honda K, Sakuma T, Kawai A, Morioka H, Toyama Y, 622 Yamada T. (2014)MicroRNA expression and functional profiles of osteosarcoma [J]. Oncology. 86: 623 94-103.

624 [30] Akiyuki Wakita, Satoru Motoyama, Yusuke Sato, Souichi Koyota, Shuetsu Usami, Kei Yoshino, 625 Tomohiko Sasaki, Kazuhiro Imai, Hajime Saito, Yoshihiro Minamiya. (2015)REG la activates c-Jun 626 through MAPK pathways to enhance the radiosensitivity of squamous esophageal cancer cells [J]. 
627 Tumour Biol. 36(7): 5249-5254.

628 [31]Dean B Reardon, Joseph N Contessa, Ross B Mikkelsen, Kristoffer Valerie, Cyrus Amir, Paul 629 Dent, Rupert K Schmidt-Ullrich. (1999)Dominant negative EGFR-CD533 and inhibition of MAPK 630 modify JNK1 activation and enhance radiation toxicity of human mammary carcinoma cells [J]. 631 Oncogene. 18(33):4756-4766.

632 [32]Wu D, Zhou W, Wang S, Zhou Z, Wang S, Chen L. (2015)Tob1 enhances radiosensitivity of 633 breast cancer cells involving the JNK and p38 pathways [J]. Cell Biol Int. 39(12):1425-1430. 